\title{
ON COMPRESSIONS OF SELF-ADJOINT EXTENSIONS OF A SYMMETRIC LINEAR RELATION
}

\author{
V.I. MOGILEVSKII
}

\begin{abstract}
Let $A$ be a symmetric linear relation in the Hilbert space $\mathfrak{H}$ with equal deficiency indices $n_{ \pm}(A) \leq \infty$. A self-adjoint linear relation $\widetilde{A} \supset A$ in some Hilbert space $\widetilde{\mathfrak{H}} \supset \mathfrak{H}$ is called an exit space extension of $A$; such an extension is called finitecodimensional if $\operatorname{dim}(\widetilde{\mathfrak{H}} \ominus \mathfrak{H})<\infty$. We study the compressions $C(\widetilde{A})=P_{\mathfrak{H}} \widetilde{A}\lceil\mathfrak{H}$ of exit space extensions $\widetilde{A}=\widetilde{A}^{*}$. For a certain class of extensions $\widetilde{A}$ we parameterize the compressions $C(\widetilde{A})$ by means of abstract boundary conditions. This enables us to characterize various properties of $C(\widetilde{A})$ (in particular, self-adjointness) in terms of the parameter for $\widetilde{A}$ in the Krein formula for resolvents. We describe also the compressions of a certain class of finite-codimensional extensions. These results develop the results by A. Dijksma and H. Langer obtained for a densely defined symmetric operator $A$ with finite deficiency indices.
\end{abstract}

\section{INTRODUCTION}

Let $\mathfrak{H}$ be a subspace in a Hilbert space $\widetilde{\mathfrak{H}}$ and let $\widetilde{A}$ be a linear relation (in particular operator) in $\widetilde{\mathfrak{H}}$. Recall that a linear relation $C(\widetilde{A})$ in $\mathfrak{H}$ given by $C(\widetilde{A})=P_{\mathfrak{H}} \widetilde{A} \uparrow \mathfrak{H}$ is called a compression of $\widetilde{A}$; moreover, the relation $\widetilde{A}$ and its compression $C(\widetilde{A})$ are called finite-codimensional if $\operatorname{dim}(\widetilde{\mathfrak{H}} \ominus \mathfrak{H})<\infty$. Compressions of linear operators or relations were recently studied in $[2,3,4,11,12,20]$. In particular, it was shown in [3] that a finite-codimensional compression $C(\widetilde{A})$ of a self-adjoint linear relation $\widetilde{A}$ is self-adjoint (for operators $\widetilde{A}=\widetilde{A}^{*}$ this fact was established earlier in [24]).

Assume now that $A$ is a not necessarily densely defined symmetric operator in a Hilbert space $\mathfrak{H}$. A self-adjoint linear relation $\widetilde{A} \supset A$ in a Hilbert space $\widetilde{\mathfrak{H}} \supset \mathfrak{H}$ is called an exit space extension of $A$. Denote by $\operatorname{Self}(A)$ the set of all exit space extensions $\widetilde{A}=\widetilde{A}^{*}$ of $A$ and by $\operatorname{Self}_{0}(A)$ the set of all $\widetilde{A} \in \operatorname{Self}(A)$ such that $\widetilde{A}$ is an operator. As is known $\operatorname{Self}(A)=\operatorname{Self}_{0}(A)$ if and only if $A$ is densely defined.

If $\widetilde{A} \in \operatorname{Self}(A)$, then the compression $C(\widetilde{A})$ of $\widetilde{A}$ is a symmetric extension of $A$. A description of all extensions $\widetilde{A} \in \operatorname{Self}(A)$ and their compressions $C(\widetilde{A})$ is an important problem in the extension theory of symmetric operators. In the paper by A.V. Shtraus [23] all extensions $\widetilde{A} \in \operatorname{Self}_{0}(A)$ of an operator $A$ with arbitrary (equal or unequal) deficiency indices $n_{ \pm}(A) \leq \infty$ are parameterized by means of holomorphic operatorfunctions $F(\lambda), \quad \lambda \in \mathbb{C}_{+}$, whose values are contractions from $\mathfrak{N}_{i}$ to $\mathfrak{N}_{-i}$ (here $\mathfrak{N}_{ \pm i}=$ $\operatorname{ker}\left(A^{*} \mp i\right)$ are defect subspaces of $\left.A\right)$. In [13] the result of [23] was extended to extensions $\widetilde{A} \in \operatorname{Self}(A)$. In $[22,25]$ the compressions $C(\widetilde{A})$ of the extensions $\widetilde{A} \in \operatorname{Self}_{0}(A)$ we described in terms of the asymptotic behaviour of the corresponding parameter $F(\lambda)$.

In the case $n_{+}(A)=n_{-}(A)$ another parametrization of the set $\operatorname{Self}(A)$ is given by the Krein formula for generalized resolvents $[15,17]$. This formula (see (2.36)) establishes a bijective correspondence $\widetilde{A}=\widetilde{A}_{\tau}$ between all relation-valued Nevanlinna functions $\tau=\tau(\lambda)(\lambda \in \mathbb{C} \backslash \mathbb{R})(\tau(\lambda)$ is a linear relation in an auxiliary Hilbert space $\mathcal{H})$ and all

2010 Mathematics Subject Classification. 47A06, 47A20, 47A56, 47B25.

Key words and phrases. Symmetric and self-adjoint linear relation (operator), exit space self-adjoint extension, compression, boundary triplet. 
extensions $\widetilde{A} \in \operatorname{Self}(A)$. The canonical self-adjoint extension $A_{0}$ of $A$ in (2.36) is called a basic extension. An operator-valued parameter $\tau=\tau(\lambda)$ in (2.36) is called rational if

$$
\tau(\lambda)=\mathcal{A}+\lambda \mathcal{B}+\sum_{j=1}^{l} \frac{1}{\alpha_{j}-\lambda} \mathcal{A}_{j}, \quad \lambda \in \mathbb{C} \backslash \mathbb{R},
$$

where $\alpha_{j} \in \mathbb{R}$ and $\mathcal{A}=\mathcal{A}^{*}, \mathcal{B} \geq 0$ and $\mathcal{A}_{j} \geq 0$ are bounded operators in $\mathcal{H}$. In the case $n_{+}(A)=n_{-}(A)<\infty$ an extension $\widetilde{A}_{\tau} \in \operatorname{Self}(A)$ is finite-codimensional if and only if a parameter $\tau$ is rational.

In the paper by H. Langer and A. Dijksma [12] the compressions $C\left(\widetilde{A}_{\tau}\right)$ of extensions $\widetilde{A}_{\tau}$ are investigated in terms of the parameter $\tau$ from the Krein formula (2.36). The main results of [12] can be formulated in the form of the following theorem.

Theorem 1.1. Assume that $A$ is a densely defined, closed symmetric operator in $\mathfrak{H}$ with finite deficiency indices $n_{+}(A)=n_{-}(A)<\infty$ and $A_{0}=A_{0}^{*}$ is the basic extension in the Krein formula (2.36). Let for simplicity a parameter $\tau=\tau(\lambda), \lambda \in \mathbb{C} \backslash \mathbb{R}$, in (2.36) be an operator-valued function, let $\widetilde{A}_{\tau} \in \operatorname{Self}_{0}(A)$ be the corresponding extension of $A$ and let $C\left(\widetilde{A}_{\tau}\right)$ be the compression of $\widetilde{A}_{\tau}$. Assume also that $\mathcal{B}_{\tau} \geq 0$ is an operator in $\mathcal{H}$ given by $\mathcal{B}_{\tau}=\lim _{y \rightarrow \infty} \frac{1}{i y} \tau(i y)$. Then:

(i) If

$$
\lim _{y \rightarrow \infty} y \operatorname{Im}(\tau(i y) h, h)=\infty, \quad h \in \mathcal{H}, \quad h \neq 0
$$

then $C\left(\widetilde{A}_{\tau}\right) \subset A_{0}$.

(ii) If (1.2) holds, then $C\left(\widetilde{A}_{\tau}\right)=A$ if and only if $\mathcal{B}_{\tau}=0$.

(iii) If $\mathcal{B}_{\tau}>0$, then $C\left(\widetilde{A}_{\tau}\right)=A_{0}$. If $(1.2)$ holds and $C\left(\widetilde{A}_{\tau}\right)=A_{0}$, then $\mathcal{B}_{\tau}>0$. Moreover, if $\tau$ is a rational parameter $(1.1)$, then $C\left(\widetilde{A}_{\tau}\right)=A_{0} \Longleftrightarrow \mathcal{B}_{\tau}>0$.

(iv) If $\tau$ is the rational parameter (1.1), then the extension $\widetilde{A}_{\tau}$ is finite-codimensional and the canonical self-adjoint extension $C\left(\widetilde{A}_{\tau}\right)$ of $A$ corresponds in the Krein formula to the self-adjoint linear relation $\tau_{\infty}$ in $\mathcal{H}$ given by

$$
\tau_{\infty}=\left\{\left\{h, P_{\text {ker } \mathcal{B}} \mathcal{A} h+h^{\prime}\right\}: h \in \operatorname{ker} \mathcal{B}, h^{\prime} \in \operatorname{ran} \mathcal{B}\right\} .
$$

It is also shown in [12] that $\tau_{\infty}$ admits the representation

$$
\tau_{\infty}=\left\{\left\{h, h^{\prime}\right\} \in \mathcal{H}^{2}: \exists h(\lambda) \in \mathcal{H}: h=\lim _{\lambda \rightarrow \infty} h(\lambda), h^{\prime}=\lim _{\lambda \rightarrow \infty} \tau(\lambda) h(\lambda)\right\} .
$$

In the present paper we study compressions of extensions $\widetilde{A} \in \operatorname{Self}(A)$ of a symmetric linear relation (in particular, not necessarily densely defined symmetric operator) $A$ with possibly infinite deficiency indices $n_{+}(A)=n_{-}(A) \leq \infty$. Our approach is based on the theory of boundary triplets and their Weyl functions (see $[9,14,18]$ and references therein). Recall $[14,18]$ that a collection $\Pi=\left\{\mathcal{H}, \Gamma_{0}, \Gamma_{1}\right\}$ consisting of an auxiliary Hilbert space $\mathcal{H}$ and linear mappings $\Gamma_{j}: A^{*} \rightarrow \mathcal{H}, j \in\{0,1\}$, is called a boundary triplet for $A^{*}$ if the mapping $\left(\Gamma_{0}, \Gamma_{1}\right)^{\top}$ is surjective and the abstract Green identity (2.15) is valid (here $A^{*}$ is the adjoint relation). If $\Pi=\left\{\mathcal{H}, \Gamma_{0}, \Gamma_{1}\right\}$ is a boundary triplet for $A^{*}$, then the equality (the abstract boundary conditions)

$$
\theta \rightarrow A_{\theta}:=\left\{\hat{f} \in A^{*}:\left\{\Gamma_{0} \hat{f}, \Gamma_{1} \hat{f}\right\} \in \theta\right\}
$$

gives a parametrization of all proper extensions $\widetilde{A}$ of $A$ (i.e., all linear relations $\widetilde{A}$ in $\mathfrak{H}$ with $A \subset \widetilde{A} \subset A^{*}$ ) in terms of linear relations $\theta$ in $\mathcal{H}$. Moreover, it was shown in $[9,18]$ that each boundary triplet $\Pi$ for $A^{*}$ gives rise to the Krein formula (2.36) with coefficients $A_{0}, \gamma(\lambda)$ and $M(\lambda)$ naturally defined in terms of $\Pi$. 
Assume that $A$ is a closed symmetric linear relation in $\mathfrak{H}$ with equal deficiency indices $n_{+}(A)=n_{-}(A) \leq \infty$ and let $\Pi=\left\{\mathcal{H}, \Gamma_{0}, \Gamma_{1}\right\}$ be a boundary triplet for $A^{*}$. In the paper we consider extensions $\widetilde{A}_{\tau} \in \operatorname{Self}(A)$ corresponding to a certain subclass of Nevanlinna parameters $\tau(\lambda)$ in (2.36). We show that the compression $C\left(\widetilde{A}_{\tau}\right)$ of $\widetilde{A}_{\tau}$ being a symmetric extension of $A$ admits the representation $C\left(\widetilde{A}_{\tau}\right)=A_{\theta_{c}}$ (see (1.5)) with a symmetric linear relation $\theta_{c}$, which in a certain sense is a limit value of $\tau(i y)$ when $y \rightarrow \infty$. This result enables us to characterise various properties of $C\left(\widetilde{A}_{\tau}\right)$ (self-adjointness, inclusion $C\left(\widetilde{A}_{\tau}\right) \subset A_{0}$, equalities $C\left(\widetilde{A}_{\tau}\right)=A$ and $\left.C\left(\widetilde{A}_{\tau}\right)=A_{0}\right)$ in terms of the parameter $\tau$. In the case $n_{ \pm}(A)<\infty$ the obtained results become valid for any Nevanlinna parameter $\tau$. This fact enables us to prove the following theorem which strengthens essentially statements (i) - (iii) of Theorem 1.1.

Theorem 1.2. Assume that $A$ is a closed symmetric linear relation in $\mathfrak{H}$ with finite deficiency indices $n_{+}(A)=n_{-}(A)<\infty$ and let all other assumptions of Theorem 1.1 be satisfied. Then:

(i') $C\left(\widetilde{A}_{\tau}\right) \subset A_{0}$ if and only if (1.2) holds.

(ii') $C\left(\widetilde{A}_{\tau}\right)=A$ if and only $\mathcal{B}_{\tau}=0$ and (1.2) holds.

(iii') $C\left(\widetilde{A}_{\tau}\right)=A_{0}$ if and only if $\mathcal{B}_{\tau}>0$.

We prove also the following theorem which characterizes extensions $\widetilde{A} \in \operatorname{Self}(A)$ with self-adjoint compression $C(\widetilde{A})$.

Theorem 1.3. Let the assumptions be the same as in Theorem 1.2. Then:

(i) $C\left(\widetilde{A}_{\tau}\right)$ is self-adjoint if and only if $\lim _{y \rightarrow \infty} y \operatorname{Im}(\tau(i y) h, h)<\infty$ for all $h \in \operatorname{ker} \mathcal{B}_{\tau}$.

(i) $C\left(\widetilde{A}_{\tau}\right)$ is self-adjoint and transversal with $A_{0}$ (that is, $C\left(\widetilde{A}_{\tau}\right) \cap A_{0}=A$ and $\left.C\left(\widetilde{A}_{\tau}\right) \widehat{+} A_{0}=A^{*}\right)$ if and only if $\lim _{y \rightarrow \infty} y \operatorname{Im}(\tau(i y) h, h)<\infty$ for all $h \in \mathcal{H}$. In this case the corresponding parameter for $C\left(\widetilde{A}_{\tau}\right)$ in the Krein formula is the operator $\mathcal{N}_{\tau}^{\prime}$ in $\mathcal{H}$ given by $\mathcal{N}_{\tau}^{\prime} h=\lim _{y \rightarrow \infty} \tau(i y) h, h \in \mathcal{H}$. Moreover, in the sense of $(1.5) C\left(\widetilde{A}_{\tau}\right)=A_{\mathcal{N}_{\tau}}$ with $\mathcal{N}_{\tau}=-\mathcal{N}_{\tau}^{\prime}$.

Finally, we extend statement (iv) of Theorem 1.1 to a certain class of finite-codimensional extensions $\widetilde{A} \in \operatorname{Self}(A)$ of a symmetric linear relation $A$ with possibly infinite equal deficiency indices $n_{ \pm}(A)$. In the case $n_{+}(A)=n_{-}(A)<\infty$ this result holds for any finite-codimensional extension $\widetilde{A} \in \operatorname{Self}(A)$.

\section{Preliminaries}

2.1. Notations. The following notations will be used throughout the paper: $\mathfrak{H}, \mathcal{H}$ denote separable Hilbert spaces; $\boldsymbol{B}\left(\mathcal{H}_{1}, \mathcal{H}_{2}\right)$ is the set of all bounded linear operators defined on $\mathcal{H}_{1}$ with values in $\mathcal{H}_{2} ; A \uparrow \mathcal{L}$ is a restriction of the operator $A \in \boldsymbol{B}\left(\mathcal{H}_{1}, \mathcal{H}_{2}\right)$ onto the linear manifold $\mathcal{L} \subset \mathcal{H}_{1} ; P_{\mathcal{L}}$ is the orthoprojection in $\mathfrak{H}$ onto the subspace $\mathcal{L} \subset \mathfrak{H}$; $\mathbb{C}_{+}\left(\mathbb{C}_{-}\right)$is the open upper (lower) half-plane of the complex plane.

Recall that a linear manifold $T$ in the Hilbert space $\mathcal{H}_{0} \oplus \mathcal{H}_{1}(\mathcal{H} \oplus \mathcal{H})$ is called a linear relation from $\mathcal{H}_{0}$ to $\mathcal{H}_{1}$ (resp. in $\mathcal{H}$ ). The set of all closed linear relations from $\mathcal{H}_{0}$ to $\mathcal{H}_{1}($ in $\mathcal{H})$ will be denoted by $\widetilde{\mathcal{C}}\left(\mathcal{H}_{0}, \mathcal{H}_{1}\right)$ (resp. $\widetilde{\mathcal{C}}(\mathcal{H})$ ). Clearly for each linear operator $T: \operatorname{dom} T \rightarrow \mathcal{H}_{1}, \operatorname{dom} T \subset \mathcal{H}_{0}$, its $\operatorname{gr} T=\{\{f, T f\}: f \in \operatorname{dom} T\}$ is a linear relation from $\mathcal{H}_{0}$ to $\mathcal{H}_{1}$. This fact enables one to consider an operator as a linear relation.

For a linear relation $T$ from $\mathcal{H}_{0}$ to $\mathcal{H}_{1}$ we denote by

$$
\begin{aligned}
& \operatorname{dom} T:=\left\{h_{0} \in \mathcal{H}_{0}: \exists h_{1} \in \mathcal{H}_{1} \quad\left\{h_{0}, h_{1}\right\} \in T\right\}, \quad \operatorname{ker} T:=\left\{h_{0} \in \mathcal{H}_{0}:\left\{h_{0}, 0\right\} \in T\right\} \\
& \operatorname{ran} T:=\left\{h_{1} \in \mathcal{H}_{1}: \exists h_{0} \in \mathcal{H}_{0} \quad\left\{h_{0}, h_{1}\right\} \in T\right\}, \quad \operatorname{mul} T:=\left\{h_{1} \in \mathcal{H}_{1}:\left\{0, h_{1}\right\} \in T\right\}
\end{aligned}
$$


the domain, kernel, range and multivalued part of $T$ respectively; moreover, we let

$$
\widehat{\operatorname{mul}} T=\{0\} \oplus \operatorname{mul} T=\left\{\left\{0, h_{1}\right\}: h_{1} \in \operatorname{mul} T\right\} .
$$

Denote also by $T^{-1}$ and $T^{*}$ the inverse and adjoint linear relations of $T$ respectively.

For an operator $T=T^{*} \in \boldsymbol{B}(\mathfrak{H})$ we write $T \geq 0$ if $(T f, f) \geq 0, f \in \mathfrak{H}$, and $T>0$ if $T-\alpha I \geq 0$ with some $\alpha>0$.

For linear relations $T_{1}$ and $T_{2}$ in $\mathcal{H}$ we let $T_{1} \widehat{+} T_{2}=\left\{\widehat{f}+\widehat{g}: \widehat{f} \in T_{1}, \widehat{g} \in T_{2}\right\}$.

2.2. Nevanlinna functions. Recall that a holomorphic operator function $M: \mathbb{C} \backslash \mathbb{R} \rightarrow$ $\boldsymbol{B}(\mathcal{H})$ is called a Nevanlinna function if $\operatorname{Im} \lambda \cdot \operatorname{Im} M(\lambda) \geq 0$ and $M^{*}(\lambda)=M(\bar{\lambda}), \quad \lambda \in \mathbb{C} \backslash \mathbb{R}$. The class of all Nevanlinna $\boldsymbol{B}(\mathcal{H})$-valued functions will be denoted by $R[\mathcal{H}]$.

Definition 2.1. The operator-function $M \in R[\mathcal{H}]$ is referred to the class:

(i) $R_{c}[\mathcal{H}]$, if ran $\operatorname{Im} M(\lambda)$ is closed for all $\lambda \in \mathbb{C} \backslash \mathbb{R}$;

(ii) $R_{u}[\mathcal{H}]$, if $(\operatorname{Im} M(\lambda))^{-1} \in \boldsymbol{B}(\mathcal{H})$ for all $\lambda \in \mathbb{C} \backslash \mathbb{R}$ or equivalently if $\operatorname{Im} \lambda \cdot \operatorname{Im} M(\lambda)>$ $0, \lambda \in \mathbb{C} \backslash \mathbb{R}$.

Clearly $R_{u}[\mathcal{H}] \subset R_{c}[\mathcal{H}] \subset R[\mathcal{H}]$; moreover in the case $\operatorname{dim} \mathcal{H}<\infty$ one has $R_{c}[\mathcal{H}]=$ $R[\mathcal{H}]$.

The following proposition is well known (see e.g. [18]).

Proposition 2.2. If $M \in R[\mathcal{H}]$, then the equality

$$
\mathcal{B}_{M}=s-\lim _{y \rightarrow \infty} \frac{1}{i y} M(i y)
$$

defines the operator $\mathcal{B}_{M} \in \boldsymbol{B}(\mathcal{H})$ such that $\mathcal{B}_{M} \geq 0$. Moreover, the equality

$$
\operatorname{dom} \mathcal{N}_{M}=\left\{h \in \mathcal{H}: \lim _{y \rightarrow \infty} y \operatorname{Im}(M(i y) h, h)<\infty\right\}
$$

defines the (not necessarily closed) linear manifold $\operatorname{dom} \mathcal{N}_{M} \subset \mathcal{H}$ and for each $h \in$ $\operatorname{dom} \mathcal{N}_{M}$ there exists the limit

$$
\mathcal{N}_{M} h:=\lim _{y \rightarrow \infty} M(i y) h, \quad h \in \operatorname{dom} \mathcal{N}_{M} .
$$

Hence the equalities (2.2) and (2.3) define the linear operator $\mathcal{N}_{M}: \operatorname{dom} \mathcal{N}_{M} \rightarrow \mathcal{H}$.

Proposition 2.3. Let $\tau \in R[\mathcal{H}]$. Then the subspace $\mathcal{H}^{\prime \prime}:=\operatorname{ker} \operatorname{Im} \tau(\lambda) \subset \mathcal{H}$ does not depend on $\lambda \in \mathbb{C} \backslash \mathbb{R}$ and the block representation

$$
\tau(\lambda)=\left(\begin{array}{cc}
\tau_{1}(\lambda) & B_{1} \\
B_{1}^{*} & B_{2}
\end{array}\right): \mathcal{H}^{\prime} \oplus \mathcal{H}^{\prime \prime} \rightarrow \mathcal{H}^{\prime} \oplus \mathcal{H}^{\prime \prime}, \quad \lambda \in \mathbb{C} \backslash \mathbb{R}
$$

holds with $\mathcal{H}^{\prime}=\mathcal{H} \ominus \mathcal{H}^{\prime \prime}, B_{2}=B_{2}^{*} \in \boldsymbol{B}\left(\mathcal{H}^{\prime \prime}\right), B_{1} \in \boldsymbol{B}\left(\mathcal{H}^{\prime \prime}, \mathcal{H}^{\prime}\right)$ and the operator-function $\tau_{1}(\cdot) \in R\left[\mathcal{H}^{\prime}\right]$ such that ker $\operatorname{Im} \tau_{1}(\lambda)=\{0\}, \lambda \in \mathbb{C} \backslash \mathbb{R}$. Moreover, $\tau \in R_{c}[\mathcal{H}]$ if and only if $\tau_{1} \in R_{u}\left[\mathcal{H}^{\prime}\right]$.

Proof. As is known the Cayley transform $K(\lambda)=(\tau(\lambda)-i)(\tau(\lambda)+i)^{-1}, \lambda \in \mathbb{C}_{+}$, is a holomorphic contractive operator-function on $\mathbb{C}_{+}$and for each $\lambda \in \mathbb{C}_{+}$the operator $\tau(\lambda)$ admits the representation

$$
f=i(K(\lambda)-I) h, \quad \tau(\lambda) f=(K(\lambda)+I) h, \quad h \in \mathcal{H} .
$$

It follows from (2.5) that

$$
\operatorname{Im}(\tau(\lambda) f, f)=\|h\|^{2}-\|K(\lambda) h\|^{2}
$$

for all $\lambda \in \mathbb{C}_{+}, h \in \mathcal{H}$ and $f=i(K(\lambda)-I) h$. According to [19] the subspace $\mathcal{H}_{0}=\{h \in$ $\mathcal{H}:\|K(\lambda) h\|=\|h\|\}$ and the operator $V=K(\lambda)\left\lceil\mathcal{H}_{0}\left(\in \boldsymbol{B}\left(\mathcal{H}_{0}, \mathcal{H}\right)\right)\right.$ do not depend on $\lambda \in \mathbb{C}_{+}$. Moreover, by (2.5) and (2.6) ker $\operatorname{Im} \tau(\lambda)=(V-I) \mathcal{H}_{0}$ and hence the subspace 
$\mathcal{H}^{\prime \prime}:=\operatorname{ker} \operatorname{Im} \tau(\lambda)$ does not depend on $\lambda \in \mathbb{C}_{+}$. Note also that by $(2.5)$ the operator $\tau(\lambda) \uparrow \mathcal{H}^{\prime \prime}$ is defined by

$$
f=i(V-I) h_{0}, \quad \tau(\lambda) f=(V+I) h_{0}, \quad h_{0} \in \mathcal{H}_{0} .
$$

Therefore $B=\tau(\lambda) \uparrow \mathcal{H}^{\prime \prime}\left(\in \boldsymbol{B}\left(\mathcal{H}^{\prime \prime}, \mathcal{H}\right)\right)$ does not depend on $\lambda \in \mathbb{C}_{+}$and, consequently, $\tau(\lambda)$ admits the block representation

$$
\tau(\lambda)=\left(\begin{array}{ll}
\tau_{1}(\lambda) & B_{1} \\
\tau_{2}(\lambda) & B_{2}
\end{array}\right): \mathcal{H}^{\prime} \oplus \mathcal{H}^{\prime \prime} \rightarrow \mathcal{H}^{\prime} \oplus \mathcal{H}^{\prime \prime}, \quad \lambda \in \mathbb{C}_{+}
$$

This implies that

$$
\operatorname{Im} \tau(\lambda)=\left(\begin{array}{cc}
\operatorname{Im} \tau_{1}(\lambda) & \frac{1}{2 i}\left(B_{1}-\tau_{2}^{*}(\lambda)\right. \\
\frac{1}{2 i}\left(\tau_{2}(\lambda)-B_{1}^{*}\right) & \operatorname{Im} B_{2}
\end{array}\right): \mathcal{H}^{\prime} \oplus \mathcal{H}^{\prime \prime} \rightarrow \mathcal{H}^{\prime} \oplus \mathcal{H}^{\prime \prime}, \quad \lambda \in \mathbb{C}_{+}
$$

and hence $\operatorname{Im} B_{2}=0, \tau_{1}(\lambda)=B_{1}^{*}$. Therefore by (2.7) the equality (2.4) holds with $B_{2}=B_{2}^{*}$, which implies that

$$
\operatorname{Im} \tau(\lambda)=\left(\begin{array}{cc}
\operatorname{Im} \tau_{1}(\lambda) & 0 \\
0 & 0
\end{array}\right): \mathcal{H}^{\prime} \oplus \mathcal{H}^{\prime \prime} \rightarrow \mathcal{H}^{\prime} \oplus \mathcal{H}^{\prime \prime}, \quad \lambda \in \mathbb{C}_{+} .
$$

Hence ker $\operatorname{Im} \tau_{1}(\lambda)=\{0\}$ and the required statements are proved for $\lambda \in \mathbb{C}_{+}$. The same statements for $\lambda \in \mathbb{C}_{-}$are implied by $\tau(\lambda)=\tau^{*}(\bar{\lambda}), \lambda \in \mathbb{C}_{-}$.

As is known (see e.g. [8]) a function $\tau: \mathbb{C} \backslash \mathbb{R} \rightarrow \widetilde{\mathcal{C}}(\mathcal{H})$ is referred to the class $\widetilde{R}(\mathcal{H})$ of Nevanlinna relation valued functions if:

(i) $\operatorname{Im}\left(f^{\prime}, f\right) \geq 0, \quad\left\{f, f^{\prime}\right\} \in \tau(\lambda), \quad \lambda \in \mathbb{C}_{+}$;

(ii) $(\tau(\lambda)+i)^{-1} \in \boldsymbol{B}(\mathcal{H}), \lambda \in \mathbb{C}_{+}$, and $(\tau(\lambda)+i)^{-1}$ is a holomorphic operator-function in $\mathbb{C}_{+}$;

(iii) $\tau^{*}(\lambda)=\tau(\bar{\lambda}), \lambda \in \mathbb{C} \backslash \mathbb{R}$.

A function $\tau \in \widetilde{R}(\mathcal{H})$ is referred to the class $R(\mathcal{H})$ if its values are operators, i.e., if $\operatorname{mul} \tau(\lambda)=0, \lambda \in \mathbb{C} \backslash \mathbb{R}$. If $\tau(\cdot) \in R(\mathcal{H})$, then $\overline{\operatorname{dom} \tau(\lambda)}=\mathcal{H}, \lambda \in \mathbb{C} \backslash \mathbb{R}$.

It is clear that $R[\mathcal{H}] \subset R(\mathcal{H}) \subset \widetilde{R}(\mathcal{H})$.

According to [15] for each function $\tau \in \widetilde{R}(\mathcal{H})$ the multivalued part $\mathcal{K}:=\operatorname{mul} \tau(\lambda)$ of $\tau(\lambda)$ does not depend on $\lambda \in \mathbb{C} \backslash \mathbb{R}$ and the decompositions

$$
\mathcal{H}=\mathcal{H}_{0} \oplus \mathcal{K}, \quad \tau(\lambda)=\tau_{0}(\lambda) \oplus \widehat{\mathcal{K}}, \quad \lambda \in \mathbb{C} \backslash \mathbb{R}
$$

hold with $\tau_{0} \in R\left[\mathcal{H}_{0}\right]$ and $\widehat{\mathcal{K}}=\widehat{\operatorname{mul}} \tau(\lambda)=\{0\} \oplus \mathcal{K}$. The operator function $\tau_{0}$ is called the operator part of $\tau$.

Definition 2.4. A relation valued function $\tau \in \widetilde{R}(\mathcal{H})$ is referred to the class $\widetilde{R}_{c}(\mathcal{H})$ $\left(\widetilde{R}_{u}(\mathcal{H})\right)$ if decompositions $(2.9)$ hold with $\tau_{0} \in R_{c}\left[\mathcal{H}_{0}\right]$ (resp. with $\tau_{0} \in R_{u}\left[\mathcal{H}_{0}\right]$ ).

Clearly $\widetilde{R}_{u}(\mathcal{H}) \subset \widetilde{R}_{c}(\mathcal{H}) \subset \widetilde{R}(\mathcal{H})$ and in the case $\operatorname{dim} \mathcal{H}<\infty$ one has $\widetilde{R}_{c}(\mathcal{H})=\widetilde{R}(\mathcal{H})$. The following corollary is immediate from Proposition 2.3.

Corollary 2.5. The relation valued function $\tau: \mathbb{C} \backslash \mathbb{R} \rightarrow \widetilde{\mathcal{C}}(\mathcal{H})$ belongs to the class $\widetilde{R}_{c}(\mathcal{H})$ if and only if there exist a decomposition

$$
\mathcal{H}=\mathcal{H}_{0} \oplus \mathcal{K}=\underbrace{\mathcal{H}^{\prime} \oplus \mathcal{H}^{\prime \prime}}_{\mathcal{H}_{0}} \oplus \mathcal{K}
$$

operators $B_{1} \in \boldsymbol{B}\left(\mathcal{H}^{\prime \prime}, \mathcal{H}^{\prime}\right), B_{2}=B_{2}^{*} \in \boldsymbol{B}\left(\mathcal{H}^{\prime \prime}\right)$ and an operator-function $\tau_{1} \in R_{u}\left[\mathcal{H}^{\prime}\right]$ such that $\tau(\lambda)$ admits the representation (2.9) with the operator function

$$
\tau_{0}(\lambda)=\left(\begin{array}{ll}
\tau_{1}(\lambda) & -B_{1} \\
-B_{1}^{*} & -B_{2}
\end{array}\right): \underbrace{\mathcal{H}^{\prime} \oplus \mathcal{H}^{\prime \prime}}_{\mathcal{H}_{0}} \rightarrow \underbrace{\mathcal{H}^{\prime} \oplus \mathcal{H}^{\prime \prime}}_{\mathcal{H}_{0}}, \quad \lambda \in \mathbb{C} \backslash \mathbb{R} .
$$


This means that

$$
\tau(\lambda)=\left\{\left\{h \oplus \varphi,\left(\tau_{1}(\lambda) h-B_{1} \varphi\right) \oplus\left(-B_{1}^{*} h-B_{2} \varphi\right) \oplus k\right\}: h \oplus \varphi \in \mathcal{H}^{\prime} \oplus \mathcal{H}^{\prime \prime}, k \in \mathcal{K}\right\} .
$$

Remark 2.6. In the following for the relation valued function $\tau \in \widetilde{R}_{c}(\mathcal{H})$ represented in accordance with Corollary 2.5 by $(2.10)$ and (2.12) we will use the notation $\tau=$ $\left\{\mathcal{H}^{\prime} \oplus \mathcal{H}^{\prime \prime} \oplus \mathcal{K}, B_{1}, B_{2}, \tau_{1}\right\}$.

2.3. Boundary triplets and Weyl function. Recall that a linear relation $T$ in $\mathcal{H}$ is called: (i) symmetric if $T \subset T^{*}$ or, equivalently, if

$$
\left(f^{\prime}, g\right)-\left(f, g^{\prime}\right)=0, \quad\left\{f, f^{\prime}\right\},\left\{g, g^{\prime}\right\} \in T
$$

(ii) self-adjoint if $T=T^{*}$ (and hence $T \in \widetilde{\mathcal{C}}(\mathcal{H})$ ).

Assume that $\theta$ is a symmetric relation in $\mathcal{H}$ with a closed multivalued part mul $\theta$. Then

$$
\mathcal{H}=\mathcal{H}_{0} \oplus \mathcal{K}, \quad \theta=B \oplus \widehat{\operatorname{mul}} \theta=\{\{h, B h+k\}: h \in \operatorname{dom} \theta, k \in \operatorname{mul} \theta\},
$$

where $\mathcal{K}=\operatorname{mul} \theta, \mathcal{H}_{0}=\mathcal{H} \ominus \operatorname{mul} \theta$ and $B$ is a symmetric operator in $\mathcal{H}_{0}$ (the operator part of $\theta$ ) with $\operatorname{dom} B=\operatorname{dom} \theta \subset \mathcal{H}_{0}$. Clearly, $\theta$ is closed (self-adjoint) if and only if $B$ is closed (self-adjoint).

The following lemma will be useful in the sequel.

Lemma 2.7. Let $\theta$ be a symmetric linear relation in $\mathcal{H}$. Then:

(i) $\operatorname{dom} \theta \subset \mathcal{H} \ominus \operatorname{mul} \theta$.

(ii) If $\overline{\operatorname{mul} \theta}=\operatorname{mul} \theta$ and $\mathcal{H} \ominus \operatorname{mul} \theta \subset \operatorname{dom} \theta$, then $\theta^{*}=\theta$.

(iii) If $\operatorname{dim} \mathcal{H}<\infty$, then the condition $\mathcal{H} \ominus \operatorname{mul} \theta \subset \operatorname{dom} \theta$ is equivalent to $\theta^{*}=\theta$.

Proof. (i) Statement (i) directly follows from (2.13)

(ii) Assume that $\overline{\operatorname{mul} \theta}=\operatorname{mul} \theta$ and $\mathcal{H} \ominus \operatorname{mul} \theta \subset \operatorname{dom} \theta$. Then in the representation (2.14) of $\theta$ one has $\operatorname{dom} B=\operatorname{dom} \theta=\mathcal{H}_{0}$. Therefore $B=B^{*} \in \boldsymbol{B}\left(\mathcal{H}_{0}\right)$ and, consequently, $\theta^{*}=\theta$.

(iii) Assume that $\operatorname{dim} \mathcal{H}<\infty$. Then mul $\theta$ is closed and hence decompositions (2.14) hold. Moreover, $\operatorname{dim} \mathcal{H}_{0}<\infty$ and therefore $\theta^{*}=\theta$ if and only if $\operatorname{dom} B=\mathcal{H}_{0}$ or, equivalently, $\operatorname{dom} \theta=\mathcal{H} \ominus \operatorname{mul} \theta$. This yields statement (ii).

In the following we denote by $A$ a closed symmetric linear relation (in particular closed not necessarily densely defined symmetric operator) in a Hilbert space $\mathfrak{H}$. Let $\mathfrak{N}_{\lambda}(A)=$ $\operatorname{ker}\left(A^{*}-\lambda\right)(\lambda \in \mathbb{C} \backslash \mathbb{R})$ be a defect subspace of $A$, let $\widehat{\mathfrak{N}}_{\lambda}(A)=\left\{\{f, \lambda f\}: f \in \mathfrak{N}_{\lambda}(A)\right\}$, let $\mathfrak{N}(A)=\mathfrak{H} \ominus \operatorname{dom} A\left(=\operatorname{mul} A^{*}\right)$ and let $n_{ \pm}(A):=\operatorname{dim} \mathfrak{N}_{\lambda}(A) \leq \infty, \lambda \in \mathbb{C}_{ \pm}$, be deficiency indices of $A$. Denote by $\operatorname{ext}(A)$ the set of all proper extensions of $A$ (i.e., the set of all relations $\widetilde{A}$ in $\mathfrak{H}$ such that $\left.A \subset \widetilde{A} \subset A^{*}\right)$ and by $\overline{\operatorname{ext}}(A)$ the set of closed extensions $\widetilde{A} \in \operatorname{ext}(A)$. Recall that two extensions $\widetilde{A}_{1}, \widetilde{A}_{2} \in \overline{\operatorname{ext}}(A)$ are called transversal if $\widetilde{A}_{1} \cap \widetilde{A}_{2}=A$ and $\widetilde{A}_{1} \widehat{+} \widetilde{A}_{2}=A^{*}$.

Definition 2.8. $[14,5]$ A collection $\Pi=\left\{\mathcal{H}, \Gamma_{0}, \Gamma_{1}\right\}$ consisting of a Hilbert space $\mathcal{H}$ and linear mappings $\Gamma_{j}: A^{*} \rightarrow \mathcal{H}, j \in\{0,1\}$, is called a boundary triplet for $A^{*}$, if the mapping $\Gamma=\left(\Gamma_{0}, \Gamma_{1}\right)^{\top}$ from $A^{*}$ into $\mathcal{H} \oplus \mathcal{H}$ is surjective and the following Green's identity holds:

$$
\left(f^{\prime}, g\right)-\left(f, g^{\prime}\right)=\left(\Gamma_{1} \widehat{f}, \Gamma_{0} \widehat{g}\right)-\left(\Gamma_{0} \widehat{f}, \Gamma_{1} \widehat{g}\right), \quad \widehat{f}=\left\{f, f^{\prime}\right\}, \widehat{g}=\left\{g, g^{\prime}\right\} \in A^{*} .
$$

Proposition 2.9. $[14,18]$ If $\Pi=\left\{\mathcal{H}, \Gamma_{0}, \Gamma_{1}\right\}$ is a boundary triplet for $A^{*}$, then $n_{+}(A)=$ $n_{-}(A)=\operatorname{dim} \mathcal{H}$. Conversely, for each symmetric relation $A$ in $\mathfrak{H}$ with equal deficiency indices $n_{+}(A)=n_{-}(A)$ there exists a boundary triplet for $A^{*}$. 
Proposition 2.10. $[14,18]$ Let $\Pi=\left\{\mathcal{H}, \Gamma_{0}, \Gamma_{1}\right\}$ be a boundary triplet for $A^{*}$. Then:

(i) $\operatorname{ker} \Gamma=A$ and $\Gamma$ is a bounded operator from $A^{*}$ onto $\mathcal{H} \oplus \mathcal{H}$.

(ii) The mapping

$$
\theta \rightarrow A_{\theta}:=\left\{\hat{f} \in A^{*}:\left\{\Gamma_{0} \hat{f}, \Gamma_{1} \hat{f}\right\} \in \theta\right\}
$$

establishes a bijective correspondence between all linear relations $\theta$ in $\mathcal{H}$ and all extensions $\widetilde{A}=A_{\theta} \in \operatorname{ext}(A)$. In the case $\theta \in \boldsymbol{B}(\mathcal{H})$ one has $A_{\theta}=\left\{\hat{f} \in A^{*}: \Gamma_{1} \hat{f}=\theta \Gamma_{0} \hat{f}\right\}$

(iii) $\left(A_{\theta}\right)^{*}=A_{\theta^{*}}$

(iv) $\bar{A}_{\theta}=A_{\bar{\theta}}$ and hence $A_{\theta} \in \overline{\operatorname{ext}}(A)$ if and only if $\theta \in \widetilde{\mathcal{C}}(\mathcal{H})$

(v) $A_{\theta}$ is symmetric (self-adjoint) if and only if $\theta$ is symmetric (resp. self-adjoint)

(vi) The equality

$$
A_{0}:=\operatorname{ker} \Gamma_{0}=\left\{\hat{f} \in A^{*}: \Gamma_{0} \hat{f}=0\right\}
$$

defines a self-adjoint extension $A_{0}$ of $A$.

(vii) The extensions $A_{0}$ and $A_{\theta} \in \overline{\operatorname{ext}}(A)$ are transversal if and only if $\theta \in \boldsymbol{B}(\mathcal{H})$.

The following lemma will be useful in the sequel.

Lemma 2.11. Assume that $\Pi=\left\{\mathcal{H}, \Gamma_{0}, \Gamma_{1}\right\}$ is a boundary triplet for $A^{*}$,

$$
\mathcal{H}=\mathcal{H}^{\prime} \oplus \mathcal{H}^{\prime \prime} \oplus \mathcal{K}
$$

and the block representations of $\Gamma_{0}$ and $\Gamma_{1}$ are

$$
\Gamma_{0}=\left(\begin{array}{l}
\Gamma_{01} \\
\Gamma_{02} \\
\Gamma_{03}
\end{array}\right): A^{*} \rightarrow \mathcal{H}^{\prime} \oplus \mathcal{H}^{\prime \prime} \oplus \mathcal{K}, \quad \Gamma_{1}=\left(\begin{array}{c}
\Gamma_{11} \\
\Gamma_{12} \\
\Gamma_{13}
\end{array}\right): A^{*} \rightarrow \mathcal{H}^{\prime} \oplus \mathcal{H}^{\prime \prime} \oplus \mathcal{K} .
$$

Moreover, let $B \in \mathcal{B}\left(\mathcal{H}^{\prime \prime}, \mathcal{H}^{\prime} \oplus \mathcal{H}^{\prime \prime}\right)$ be the operator with the block representation

$$
B=\left(\begin{array}{l}
B_{1} \\
B_{2}
\end{array}\right): \mathcal{H}^{\prime \prime} \rightarrow \mathcal{H}^{\prime} \oplus \mathcal{H}^{\prime \prime}
$$

such that $B_{2}=B_{2}^{*}$ (this means that $B$ is a bounded symmetric operator in $\mathcal{H}^{\prime} \oplus \mathcal{H}^{\prime \prime}$ with the domain $\operatorname{dom} B=\mathcal{H}^{\prime \prime}$ ) and let $\theta_{0} \in \widetilde{\mathcal{C}}(\mathcal{H})$ be given by

$$
\theta_{0}=B \oplus \widehat{\mathcal{K}}=\left\{\left\{h, B_{1} h \oplus B_{2} h \oplus k\right\}: h \in \mathcal{H}^{\prime \prime}, k \in \mathcal{K}\right\}
$$

with $\widehat{\mathcal{K}}=\widehat{\operatorname{mul}} \theta_{0}=\{0\} \oplus \mathcal{K}$. Then:

(i) The equalities

$$
\begin{gathered}
S:=A_{\theta_{0}}=\left\{\widehat{f} \in A^{*}: \Gamma_{01} \widehat{f}=0, \Gamma_{03} \widehat{f}=0, \Gamma_{11} \widehat{f}=B_{1} \Gamma_{02} \widehat{f}, \Gamma_{12} \widehat{f}=B_{2} \Gamma_{02} \widehat{f}\right\} \\
S^{*}=\left\{\widehat{f} \in A^{*}: \Gamma_{03} \widehat{f}=0, \Gamma_{12} \widehat{f}=B_{1}^{*} \Gamma_{01} \widehat{f}+B_{2} \Gamma_{02} \widehat{f}\right\}
\end{gathered}
$$

define a symmetric extension $S \in \overline{\operatorname{ext}}(A)$ and its adjoint $S^{*}$.

(ii) The collection $\Pi^{\prime}=\left\{\mathcal{H}^{\prime}, \Gamma_{0}^{\prime}, \Gamma_{1}^{\prime}\right\}$ with operators $\Gamma_{j}^{\prime}: S^{*} \rightarrow \mathcal{H}^{\prime}$ defined by

$$
\Gamma_{0}^{\prime} \widehat{f}=\Gamma_{01} \widehat{f}, \quad \Gamma_{1}^{\prime} \widehat{f}=\Gamma_{11} \widehat{f}-B_{1} \Gamma_{02} \widehat{f}, \quad \widehat{f} \in S^{*}
$$

is a boundary triplet for $S^{*}$.

(iii) Let $\theta$ be a linear relation in $\mathcal{H}^{\prime}$ defined by

$$
\theta=\theta^{\prime} \dot{+} \widehat{\operatorname{mul}} \theta=\left\{\left\{h, \theta^{\prime} h+h^{\prime}\right\}: h \in \operatorname{dom} \theta^{\prime}, h^{\prime} \in \operatorname{mul} \theta\right\},
$$

with a linear operator $\theta^{\prime}: \operatorname{dom} \theta^{\prime} \rightarrow \mathcal{H}^{\prime}\left(\operatorname{dom} \theta^{\prime} \subset \mathcal{H}^{\prime}\right)$ and let the extension $\widetilde{A} \in \operatorname{ext}(S)$ be given by $\widetilde{A}=S_{\theta}$ (in the triplet $\left.\Pi^{\prime}\right)$. Moreover, let $\widetilde{\theta}^{\prime}: \operatorname{dom} \theta^{\prime} \oplus \mathcal{H}^{\prime \prime} \rightarrow \mathcal{H}^{\prime} \oplus \mathcal{H}^{\prime \prime}$ be a linear operator, defined by

$$
\widetilde{\theta^{\prime}}=\left(\begin{array}{cc}
\theta^{\prime} & B_{1} \\
B_{1}^{*}\left\lceil\operatorname{dom} \theta^{\prime}\right. & B_{2}
\end{array}\right): \operatorname{dom} \theta^{\prime} \oplus \mathcal{H}^{\prime \prime} \rightarrow \mathcal{H}^{\prime} \oplus \mathcal{H}^{\prime \prime}
$$


and let $\widetilde{\theta}$ be a linear relation in $\mathcal{H}$ given by

$$
\begin{gathered}
\operatorname{mul} \widetilde{\theta}=\operatorname{mul} \theta \oplus \mathcal{K} \\
\widetilde{\theta}:=\widetilde{\theta}^{\prime}+\widehat{\operatorname{mul}} \widetilde{\theta}=\left\{\left\{h \oplus \varphi,\left(\theta^{\prime} h+B_{1} \varphi+h^{\prime}\right) \oplus\left(B_{1}^{*} h+B_{2} \varphi\right) \oplus k\right\}:\right. \\
\left.h \oplus \varphi \in \operatorname{dom} \theta^{\prime} \oplus \mathcal{H}^{\prime \prime}, h^{\prime} \in \operatorname{mul} \theta, k \in \mathcal{K}\right\} .
\end{gathered}
$$

Then $\widetilde{A} \in \operatorname{ext}(A)$ and $\widetilde{A}=A_{\widetilde{\theta}}$ (in the triplet $\Pi$ ).

If in addition $\theta \in \boldsymbol{B}\left(\mathcal{H}^{\prime}\right)$, then

$$
\widetilde{\theta^{\prime}}=\left(\begin{array}{cc}
\theta & B_{1} \\
B_{1}^{*} & B_{2}
\end{array}\right): \mathcal{H}^{\prime} \oplus \mathcal{H}^{\prime \prime} \rightarrow \mathcal{H}^{\prime} \oplus \mathcal{H}^{\prime \prime}
$$

and (2.26) takes the form

$\left.(2.27) \widetilde{\theta}=\widetilde{\theta}^{\prime} \oplus \widehat{\mathcal{K}}=\left\{h \oplus \varphi,\left(\theta+B_{1} \varphi\right) \oplus\left(B_{1}^{*} h+B_{2} \varphi\right) \oplus k\right\}: h \oplus \varphi \in \mathcal{H}^{\prime} \oplus \mathcal{H}^{\prime \prime}, k \in \mathcal{K}\right\}$

Proof. (i) Clearly the relation $\theta_{0}$ is symmetric and by Proposition 2.10, (v) $S:=A_{\theta_{0}} \in$ $\overline{\operatorname{ext}}(A)$ is symmetric. Moreover, by (2.19) the second equality in (2.22) holds. Since obviously

$$
\theta_{0}^{*}=\left\{\left\{h_{1} \oplus h_{2}, h^{\prime} \oplus\left(B_{1}^{*} h_{1}+B_{2} h_{2}\right) \oplus k\right\}: h_{1} \oplus h_{2} \in \mathcal{H}^{\prime} \oplus \mathcal{H}^{\prime \prime}, h^{\prime} \in \mathcal{H}^{\prime}, k \in \mathcal{K}\right\}
$$

and by Proposition 2.10, (iii) $S^{*}=A_{\theta_{0}^{*}}$, the equality (2.23) is valid.

(ii) Let $\widehat{f}=\left\{f, f^{\prime}\right\}, \widehat{g}=\left\{g, g^{\prime}\right\} \in S^{*}$. By using Green identity (2.15) (for the triplet ПI) and (2.23) one obtains

$$
\begin{gathered}
\left(f^{\prime}, g\right)-\left(f, g^{\prime}\right)=\left(\Gamma_{11} \widehat{f}, \Gamma_{01} \widehat{g}\right)-\left(\Gamma_{01} \widehat{f}, \Gamma_{11} \widehat{g}\right)+\left(\Gamma_{12} \widehat{f}, \Gamma_{02} \widehat{g}\right)-\left(\Gamma_{02} \widehat{f}, \Gamma_{12} \widehat{g}\right)+ \\
\left(\Gamma_{13} \widehat{f}, \Gamma_{03} \widehat{g}\right)-\left(\Gamma_{03} \widehat{f}, \Gamma_{13} \widehat{g}\right)=\left(\Gamma_{11} \widehat{f}, \Gamma_{01} \widehat{g}\right)-\left(\Gamma_{01} \widehat{f}, \Gamma_{11} \widehat{g}\right)+\left(\Gamma_{01} \widehat{f}, B_{1} \Gamma_{02} \widehat{g}\right)+ \\
\left(B_{2} \Gamma_{02} \widehat{f}, \Gamma_{02} \widehat{g}\right)-\left(B_{1} \Gamma_{02} \widehat{f}, \Gamma_{01} \widehat{g}\right)-\left(\Gamma_{02} \widehat{f}, B_{2} \Gamma_{02} \widehat{g}\right)=\left(\left(\Gamma_{11}-B_{1} \Gamma_{02}\right) \widehat{f}, \Gamma_{01} \widehat{g}\right)- \\
\left(\Gamma_{01} \widehat{f},\left(\Gamma_{11}-B_{1} \Gamma_{02}\right) \widehat{g}\right)=\left(\Gamma_{1}^{\prime} \widehat{f}, \Gamma_{0}^{\prime} \widehat{g}\right)-\left(\Gamma_{0}^{\prime} \widehat{f}, \Gamma_{1}^{\prime} \widehat{g}\right) .
\end{gathered}
$$

This proves Green's identity (2.15) for operators $\Gamma_{0}^{\prime}$ and $\Gamma_{1}^{\prime}$.

Next assume that $h_{1}^{\prime}, h_{2}^{\prime} \in \mathcal{H}^{\prime}$. Since the mapping $\Gamma=\left(\Gamma_{0}, \Gamma_{1}\right)^{\top}$ is surjective, there is $\widehat{f} \in A^{*}$ such that

$$
\Gamma_{01} \widehat{f}=h_{1}^{\prime}, \quad \Gamma_{02} \widehat{f}=0, \quad \Gamma_{03} \widehat{f}=0, \quad \Gamma_{11} \widehat{f}=h_{2}^{\prime}, \quad \Gamma_{12} \widehat{f}=B_{1}^{*} h_{1}^{\prime}, \quad \Gamma_{13} \widehat{f}=0 .
$$

Hence $\Gamma_{12} \widehat{f}=B_{1}^{*} \Gamma_{01} \widehat{f}+B_{2} \Gamma_{02} \widehat{f}$ and by (2.23) $\widehat{f} \in S^{*}$. Moreover, in view of (2.24) one has $\Gamma_{0}^{\prime} \widehat{f}=h_{1}^{\prime}, \Gamma_{1}^{\prime} \widehat{f}=h_{2}^{\prime}$, which proves surjectivity of the mapping $\Gamma^{\prime}=\left(\Gamma_{0}^{\prime}, \Gamma_{1}^{\prime}\right)^{\top}$.

(iii) It follows from (2.23) and (2.24) that $\widehat{f} \in \widetilde{A}$ if and only if $\widehat{f} \in A^{*}$ and

$$
\Gamma_{01} \widehat{f} \in \operatorname{dom} \theta, \quad \Gamma_{11} \widehat{f}-B_{1} \Gamma_{02} \widehat{f}=\theta^{\prime} \Gamma_{01} \widehat{f}+h^{\prime}, \quad \Gamma_{12} \widehat{f}=B_{1}^{*} \Gamma_{01} \widehat{f}+B_{2} \Gamma_{02} \widehat{f}, \quad \Gamma_{03} \widehat{f}=0
$$

with some $h^{\prime} \in \operatorname{mul} \theta$. In turn, these conditions are equivalent to inclusions $\widehat{f} \in A^{*}$ and $\left\{\Gamma_{0} \widehat{f}, \Gamma_{1} \widehat{f}\right\} \in \widetilde{\theta}$. Hence $\widetilde{A}=A_{\widetilde{\theta}}$ (in the triplet $\Pi$ ).

Lemma 2.12. Let $L_{1}$ and $L_{2}$ be closed subspaces in a Hilbert space $\mathcal{H}$ such that $L_{1} \cap L_{2}=$ $\{0\}$. Then $\overline{L_{1} \dot{+} L_{2}}=L_{1} \dot{+} L_{2}$ if and only if there exists a closed subspace $L_{1}^{\prime} \supset L_{1}$ in $\mathcal{H}$ such that $\mathcal{H}=L_{1}^{\prime} \dot{+} L_{2}$.

Proof. Let $\overline{L_{1} \dot{+} L_{2}}=L_{1} \dot{+} L_{2}$. Then $\mathcal{H}=\left(L_{1} \dot{+} L_{2}\right) \oplus \mathcal{H}^{\prime}$ with $\mathcal{H}^{\prime}=\mathcal{H} \ominus\left(L_{1} \dot{+} L_{2}\right)$ and hence $\mathcal{H}=L_{1}^{\prime} \oplus L_{2}$ with a closed subspace $L_{1}^{\prime}=L_{1} \oplus \mathcal{H}^{\prime}$. Conversely, let $L_{1}^{\prime} \supset L_{1}$ be a closed subspace in $\mathcal{H}$ such that $\mathcal{H}=L_{1}^{\prime} \dot{+} L_{2}$ and let $\pi_{1} \in \boldsymbol{B}\left(\mathcal{H}, L_{1}^{\prime}\right)$ and $\pi_{2} \in \boldsymbol{B}\left(\mathcal{H}, L_{2}\right)$ be the corresponding skew projections onto $L_{1}^{\prime}$ and $L_{2}$ respectively. Assume that $f_{n} \in L_{1} \dot{+} L_{2}$ and $f_{n} \rightarrow f$. Then $\pi_{1} f_{n} \in L_{1}, \pi_{1} f_{n} \rightarrow \pi_{1} f$ and hence $\pi_{1} f \in L_{1}$. Since $\pi_{2} f \in L_{2}$, it follows that $f\left(=\pi_{1} f+\pi_{2} f\right) \in L_{1} \dot{+} L_{2}$. Therefore $L_{1} \dot{+} L_{2}$ is closed. 
ON COMPRESSIONS OF SELF-ADJOINT EXTENSIONS

Proposition 2.13. Let $\Pi=\left\{\mathcal{H}, \Gamma_{0}, \Gamma_{1}\right\}$ be a boundary triplet for $A^{*}$, let $A_{0}=\operatorname{ker} \Gamma_{0}$ and let $\theta \in \widetilde{\mathcal{C}}(\mathcal{H})$ be a linear relation such that decompositions (2.14) hold with a (closed) linear operator $B: \operatorname{dom} \theta \rightarrow \mathcal{H}_{0}, \operatorname{dom} \theta \subset \mathcal{H}_{0}$ (in particular, this assumption is satisfied for symmetric $\theta)$. Then $\overline{A_{\theta} \widehat{+} A_{0}}=A_{\theta} \widehat{+} A_{0}$ if and only if $B \in \boldsymbol{B}\left(\operatorname{dom} \theta, \mathcal{H}_{0}\right)$ (that is, if and only if $\overline{\operatorname{dom} \theta}=\operatorname{dom} \theta$ ).

Proof. Let $\widehat{\mathcal{H}}=\{0\} \oplus \mathcal{H}$ and $\widehat{\mathcal{H}}_{0}=\{0\} \oplus \mathcal{H}_{0}$. Since $A_{0}=A_{\widehat{\mathcal{H}}}$, it follows from Proposition 2.10 , (i) that

$$
\overline{A_{\theta} \widehat{+} A_{0}}=A_{\theta} \widehat{+} A_{0} \Longleftrightarrow \overline{\theta \widehat{+} \widehat{\mathcal{H}}}=\theta \widehat{+} \widehat{\mathcal{H}}
$$

Moreover, $B \cap \widehat{\mathcal{H}}_{0}=\{0\}$ and $\widehat{\mathcal{H}}=\widehat{\mathcal{H}}_{0} \oplus \widehat{\mathcal{K}}$, which in view of (2.14) implies that $\theta \widehat{+} \widehat{\mathcal{H}}=$ $\left(B+\widehat{\mathcal{H}}_{0}\right) \oplus \widehat{\mathcal{K}}$. Therefore

$$
\overline{\theta \widehat{+} \widehat{\mathcal{H}}}=\theta \widehat{+} \widehat{\mathcal{H}} \Longleftrightarrow \overline{B \dot{+} \widehat{\mathcal{H}}_{0}}=B \dot{+} \widehat{\mathcal{H}}_{0} .
$$

Next, by Lemma $2.12 \overline{B \dot{+} \widehat{\mathcal{H}}_{0}}=B \dot{+} \widehat{\mathcal{H}}_{0}$ if and only if there exists $B^{\prime} \in \widetilde{\mathcal{C}}\left(\mathcal{H}_{0}\right)$ such that $B \subset B^{\prime}$ and $B^{\prime} \oplus \widehat{\mathcal{H}}_{0}=\mathcal{H}_{0}^{2}$. Since the last equality is equivalent to the inclusion $B^{\prime} \in \boldsymbol{B}\left(\mathcal{H}_{0}\right)$, the following equivalence holds:

$$
\overline{B \dot{+} \widehat{\mathcal{H}}_{0}}=B \dot{+} \widehat{\mathcal{H}}_{0} \Longleftrightarrow B \in \boldsymbol{B}\left(\operatorname{dom} \theta, \mathcal{H}_{0}\right) .
$$

Now combining (2.28), (2.29) and (2.30), one obtains the required statement.

Corollary 2.14. Let $\Pi=\left\{\mathcal{H}, \Gamma_{0}, \Gamma_{1}\right\}$ be a boundary triplet for $A^{*}$, let $A_{0}=\operatorname{ker} \Gamma_{0}$ and let $\theta \in \widetilde{\mathcal{C}}(\mathcal{H})$. Then $A_{\theta} \cap A_{0}=A$ and $\overline{A_{\theta} \widehat{+} A_{0}}=A_{\theta} \widehat{+} A_{0}$ if and only if $\theta \in \boldsymbol{B}(\operatorname{dom} \theta, \mathcal{H})$.

Proof. Since $A_{0}=A_{\{0\} \oplus \mathcal{H}}$, it follows from Proposition 2.10, (i) that $A_{\theta} \cap A_{0}=A$ if and only if $\theta \cap(\{0\} \oplus \mathcal{H})=\{0\}$, that is if and only if $\theta$ is an operator. Thus $A_{\theta} \cap A_{0}=A$ if and only if decompositions (2.9) hold with $\mathcal{K}=\{0\}$. This and Proposition 2.13 yield the result.

In the rest of this subsection we recall some definitions and results from $[9,18,10]$.

Proposition 2.15. Let $\Pi=\left\{\mathcal{H}, \Gamma_{0}, \Gamma_{1}\right\}$ be a boundary triplet for $A^{*}$ and let $A_{0}=A_{0}^{*} \in$ $\overline{\operatorname{ext}}(A)$ be given by (2.17). Moreover, let $\pi_{1}$ be the orthoprojection in $\mathfrak{H} \oplus \mathfrak{H}$ onto $\mathfrak{H} \oplus\{0\}$. Then the operator $\Gamma_{0}\left\lceil\widehat{\mathfrak{N}}_{\lambda}(A), \lambda \in \mathbb{C} \backslash \mathbb{R}\right.$, isomorphically maps $\widehat{\mathfrak{N}}_{\lambda}(A)$ onto $\mathcal{H}$ and hence the equalities

$$
\gamma(\lambda)=\pi_{1}\left(\Gamma_{0}\left\lceil\widehat{\mathfrak{N}}_{\lambda}(A)\right)^{-1}, \quad \Gamma_{1}\left\lceil\widehat{\mathfrak{N}}_{\lambda}(A)=M(\lambda) \Gamma_{0}\left\lceil\widehat{\mathfrak{N}}_{\lambda}(A), \quad \lambda \in \mathbb{C} \backslash \mathbb{R}\right.\right.\right.
$$

correctly define the operator functions $\gamma(\cdot): \mathbb{C} \backslash \mathbb{R} \rightarrow \boldsymbol{B}(\mathcal{H}, \mathfrak{H})$ and $M(\cdot): \mathbb{C} \backslash \mathbb{R} \rightarrow \boldsymbol{B}(\mathcal{H})$. Moreover, $\gamma(\cdot)$ and $M(\cdot)$ satisfy the identities

$$
\begin{gathered}
\gamma(\lambda)=\gamma(z)+(\lambda-z)\left(A_{0}-\lambda\right)^{-1} \gamma(z) \\
M(z)-M^{*}(\lambda)=(z-\bar{\lambda}) \gamma^{*}(\lambda) \gamma(z), \quad z, \lambda \in \mathbb{C} \backslash \mathbb{R}
\end{gathered}
$$

which imply that $\gamma(\cdot)$ and $M(\cdot)$ are holomorphic in $\mathbb{C} \backslash \mathbb{R}$ and $M(\cdot) \in R[\mathcal{H}]$.

Definition 2.16. The operator-functions $\gamma(\cdot)$ and $M(\cdot)$ defined in Proposition 2.15 are called the $\gamma$-field and the Weyl function of the triplet $\Pi$ respectively.

Remark 2.17. It follows from (2.31) and (2.32) that $\gamma(\cdot)$ and $M(\cdot)$ are the $\gamma$-field and the $Q$-function of the pair $\left(A, A_{0}\right)$ respectively in the sense of $[16,17]$.

Definition 2.18. The (not necessarily closed) linear relation $\mathcal{F}$ in $\mathcal{H}$ defined by

$$
\mathcal{F}:=\widehat{\Gamma \operatorname{mul}} A^{*}=\left\{\left\{\Gamma_{0}\{0, n\}, \Gamma_{1}\{0, n\}\right\}: n \in \mathfrak{N}(A)\right\}
$$

is called a forbidden relation of the boundary triplet $\Pi=\left\{\mathcal{H}, \Gamma_{0}, \Gamma_{1}\right\}$ for $A^{*}$. 
In the following theorem the forbidden relation $\mathcal{F}$ is characterized in terms of the asymptotic behavior of the Weyl function.

Theorem 2.19. Let $\Pi=\left\{\mathcal{H}, \Gamma_{0}, \Gamma_{1}\right\}$ be a boundary triplet for $A^{*}$, let $M(\cdot)$ be the Weyl function of $\Pi$ and let $B_{M}$ and $\mathcal{N}_{M}$ be the operators defined by (2.1) and (2.2), (2.3) respectively. Moreover, let $\mathcal{F}$ be the forbidden relation of $\Pi$. Then

$$
\operatorname{ran} B_{M} \subset \operatorname{mul} \mathcal{F}, \quad \overline{\operatorname{ran} B_{M}}=\overline{\operatorname{mul} \mathcal{F}},
$$

and $\mathcal{F}$ admits the representation

$$
\mathcal{F}=\mathcal{N}_{M}+\widehat{\operatorname{mul}} \mathcal{F}=\left\{\left\{h, \mathcal{N}_{M} h+h^{\prime}\right\}: h \in \operatorname{dom} \mathcal{N}_{M}, h^{\prime} \in \operatorname{mul} \mathcal{F}\right\} .
$$

2.4. Exit space extensions and formula for generalized resolvents. As is known a linear relation $\widetilde{A}=\widetilde{A}^{*}$ in a Hilbert space $\widetilde{\mathfrak{H}} \supset \mathfrak{H}$ is called an exit space extension of $A$ if $A \subset \widetilde{A}$ and the minimality condition $\overline{\operatorname{span}}\left\{\mathfrak{H},(\widetilde{A}-\lambda)^{-1} \mathfrak{H}: \lambda \in \mathbb{C} \backslash \mathbb{R}\right\}=\widetilde{\mathfrak{H}}$ is satisfied. For an exit space extension $\widetilde{A} \in \widetilde{\mathcal{C}}(\widetilde{\mathfrak{H}})$ of $A$ the compressed resolvent

$$
R(\lambda)=P_{\mathfrak{H}}(\widetilde{A}-\lambda)^{-1}\lceil\mathfrak{H}, \quad \lambda \in \mathbb{C} \backslash \mathbb{R}
$$

is called a generalized resolvent of $A$ (here $P_{\mathfrak{H}}$ is the orthoprojection in $\widetilde{\mathfrak{H}}$ onto $\left.\mathfrak{H}\right)$. If two exit space extensions $\widetilde{A}_{1} \in \widetilde{\mathcal{C}}\left(\widetilde{\mathfrak{H}}_{1}\right)$ and $\widetilde{A}_{2} \in \widetilde{\mathcal{C}}\left(\widetilde{\mathfrak{H}}_{2}\right)$ of $A$ generates the same generalized resolvent $R(\lambda)$, then $\widetilde{A}_{1}$ and $\widetilde{A}_{2}$ are equivalent. The latter means that there exists a unitary operator $V \in \boldsymbol{B}\left(\widetilde{\mathfrak{H}}_{1} \ominus \mathfrak{H}, \widetilde{\mathfrak{H}}_{2} \ominus \mathfrak{H}\right)$ such that $\widetilde{A}_{2}=\widetilde{U} \widetilde{A}_{1}$ with the unitary operator $\widetilde{U}=\left(I_{\mathfrak{H}} \oplus V\right) \oplus\left(I_{\mathfrak{H}} \oplus V\right) \in B\left(\widetilde{\mathfrak{H}}_{1}^{2}, \widetilde{\mathfrak{H}}_{2}^{2}\right)$. Hence each exit space extension $\widetilde{A}$ of $A$ is defined by the generalized resolvent (2.35) uniquely up to the equivalence.

Theorem 2.20. $[9,18]$ Assume that $\Pi=\left\{\mathcal{H}, \Gamma_{0}, \Gamma_{1}\right\}$ is a boundary triplet for $A^{*}$, $A_{0}=\operatorname{ker} \Gamma_{0}$ and $\gamma(\cdot)$ and $M(\cdot)$ are the $\gamma$-field and the Weyl function of $\Pi$ respectively. Then: (i) the equality (Krein formula for generalized resolvents)

$$
P_{\mathfrak{H}}\left(\widetilde{A}_{\tau}-\lambda\right)^{-1}\left\lceil\mathfrak{H}=\left(A_{0}-\lambda\right)^{-1}-\gamma(\lambda)(\tau(\lambda)+M(\lambda))^{-1} \gamma^{*}(\bar{\lambda}), \quad \lambda \in \mathbb{C} \backslash \mathbb{R}\right.
$$

establishes a bijective correspondence $\widetilde{A}=\widetilde{A}_{\tau}$ between all relation valued functions $\tau=$ $\tau(\lambda) \in \widetilde{R}(\mathcal{H})$ and all exit space self-adjoint extensions $\widetilde{A}$ of $A$. Moreover, for each $\tau \in \widetilde{R}(\mathcal{H})$ the following equality holds:

$$
P_{\mathfrak{H}}\left(\widetilde{A}_{\tau}-\lambda\right)^{-1}\left\lceil\mathfrak{H}=\left(A_{-\tau(\lambda)}-\lambda\right)^{-1}, \quad \lambda \in \mathbb{C} \backslash \mathbb{R}\right.
$$

(ii) an extension $\widetilde{A}_{\tau}$ is canonical (that is, $\widetilde{A}_{\tau} \in \widetilde{\mathcal{C}}(\mathfrak{H})$ ) if and only if $\tau(\lambda) \equiv \theta\left(=\theta^{*}\right), \lambda \in$ $\mathbb{C} \backslash \mathbb{R}$. In this case $\widetilde{A}_{\tau}=A_{-\theta}$ (in the sense of Proposition 2.10, (ii)).

It follows from Theorem 2.20 that Krein formula (2.36) gives a parametrization $\widetilde{A}=\widetilde{A}_{\tau}$ of all exit space extensions $\widetilde{A}=\widetilde{A}^{*}$ of $A$ in terms of functions $\tau(\cdot) \in \widetilde{R}(\mathcal{H})$. Without connection with boundary triplets formula (2.36) was originally proved in $[15,17]$.

\section{Compressions of EXIT SPACE SELF-ADJOINT EXTENSIONS}

3.1. Parametrization of compressions. Assume that $\widetilde{\mathfrak{H}} \supset \mathfrak{H}$ is a Hilbert space, $\mathfrak{H}_{r}:=$ $\widetilde{\mathfrak{H}} \ominus \mathfrak{H}, P_{\mathfrak{H}}$ is the orthoprojection in $\widetilde{\mathfrak{H}}$ onto $\mathfrak{H}$ and $\widetilde{A}=\widetilde{A}^{*} \in \widetilde{\mathcal{C}}(\widetilde{\mathfrak{H}})$ is an exit space extension of $A$. In the following we let

$$
\begin{gathered}
S(\widetilde{A}):=\widetilde{A} \cap \mathfrak{H}^{2}=\left\{\widehat{f} \in \mathfrak{H}^{2}: \widehat{f} \in \widetilde{A}\right\} \\
T(\widetilde{A}):=\left\{\left\{P_{\mathfrak{H}} f, P_{\mathfrak{H}} f^{\prime}\right\}:\left\{f, f^{\prime}\right\} \in \widetilde{A}\right\} \\
C(\widetilde{A}):=P_{\mathfrak{H}} \widetilde{A}\left\lceil\mathfrak{H}=\left\{\left\{f, f^{\prime}\right\} \in \mathfrak{H}^{2}:\left\{f, f^{\prime} \oplus f_{r}^{\prime}\right\} \in \widetilde{A} \text { with some } f_{r}^{\prime} \in \mathfrak{H}_{r}\right\}\right.
\end{gathered}
$$


Clearly, $S(\widetilde{A})$ is a closed symmetric relation in $\mathfrak{H}, C(\widetilde{A})$ is a symmetric relation in $\mathfrak{H}$, $T(\widetilde{A})$ is a linear relation in $\mathfrak{H}$ and

$$
A \subset S(\widetilde{A}) \subset C(\widetilde{A}) \subset T(\widetilde{A}) \subset A^{*}
$$

Moreover, $S(\widetilde{A}) \subset \widetilde{A}$ and according to $[21,7](S(\widetilde{A}))^{*}=\overline{T(\widetilde{A})}$.

Definition 3.1. The linear relation $C(\widetilde{A})$ is called the compression of $\widetilde{A}$.

The following theorem directly follows from the results of $[6,8]$.

Theorem 3.2. Let $\Pi=\left\{\mathcal{H}, \Gamma_{0}, \Gamma_{1}\right\}$ be a boundary triplet for $A^{*}$, let $\tau \in \widetilde{R}(\mathcal{H})$ and let $\widetilde{A}_{\tau}=\widetilde{A}_{\tau}^{*}$ be the corresponding exit space extension of $A$. Then the equalities $S(\widetilde{A})=A$ and $\overline{T(\widetilde{A})}=T(\widetilde{A})$ hold if and only if $\tau \in R_{u}[\mathcal{H}]$.

Lemma 3.3. Let the assumptions of Lemma 2.11 be satisfied. Moreover, let $S \in \overline{\operatorname{ext}}(A)$ be symmetric extension (2.22) of $A$, let $\Pi^{\prime}=\left\{\mathcal{H}^{\prime}, \Gamma_{0}^{\prime}, \Gamma_{1}^{\prime}\right\}$ be boundary triplet (2.24) for $S^{*}$, let $\tau_{1}(\cdot) \in R_{u}\left[\mathcal{H}^{\prime}\right]$ and let $\widetilde{A}=\widetilde{S}_{\tau_{1}}$ be the corresponding exit space self-adjoint extension of $S$. Assume also that $\tau=\left\{\mathcal{H}^{\prime} \oplus \mathcal{H}^{\prime \prime} \oplus \mathcal{K}, B_{1}, B_{2}, \tau_{1}\right\} \in \widetilde{R}_{c}(\mathcal{H})$ (see Remark 2.6). Then $\widetilde{A} \supset A$ and $\widetilde{A}=\widetilde{A}_{\tau}$ (in the triplet $\Pi$ ).

Proof. According to (2.37)

$$
P_{\mathfrak{H}}(\widetilde{A}-\lambda)^{-1}\left\lceil\mathfrak{H}=(\widetilde{A}(\lambda)-\lambda)^{-1}, \quad \lambda \in \mathbb{C} \backslash \mathbb{R},\right.
$$

where $\widetilde{A}(\lambda)=S_{-\tau_{1}(\lambda)}$ (in the triplet $\Pi^{\prime}$ ). Moreover,

$$
\left.-\tau(\lambda)=\left\{h \oplus \varphi,\left(-\tau_{1}(\lambda) h+B_{1} \varphi\right) \oplus\left(B_{1}^{*} h+B_{2} \varphi\right) \oplus k\right\}: h \oplus \varphi \in \mathcal{H}^{\prime} \oplus \mathcal{H}^{\prime \prime}, k \in \mathcal{K}\right\} .
$$

Comparing this equality with (2.27) we obtain from Lemma 2.11, (iii) that $\widetilde{A}(\lambda) \in \overline{\operatorname{ext}}(A)$ and $\widetilde{A}(\lambda)=A_{-\tau(\lambda)}$ (in the triplet $\Pi$ ). Therefore by (3.5) and (2.37) $\widetilde{A}=\widetilde{A}_{\tau}$ (in the triplet ПI).

Proposition 3.4. Let $\Pi=\left\{\mathcal{H}, \Gamma_{0}, \Gamma_{1}\right\}$ be a boundary triplet for $A^{*}$, let $\tau \in \widetilde{R}_{c}(\mathcal{H})$ and let $\widetilde{A}_{\tau}=\widetilde{A}_{\tau}^{*}$ be the corresponding exit space extension of $A$. Assume also that

$$
\tau=\left\{\mathcal{H}^{\prime} \oplus \mathcal{H}^{\prime \prime} \oplus \mathcal{K}, B_{1}, B_{2}, \tau_{1}\right\}
$$

(see Remark 2.6), $\Gamma_{0}$ and $\Gamma_{1}$ have the block representations $(2.19)$ and $\theta_{0} \in \widetilde{\mathcal{C}}(\mathcal{H})$ is given by (2.21). Then the extension $S=S\left(\widetilde{A}_{\tau}\right)$ admits the representation (2.22) (in the triplet ПI).

Proof. Clearly the assumptions of Lemma 2.11 are satisfied with the subspaces $\mathcal{H}^{\prime}, \mathcal{H}^{\prime \prime}, \mathcal{K}$ and the operator $B=\left(B_{1}, B_{2}\right)^{\top}$. Let $S \in \overline{\operatorname{ext}}(A)$ be symmetric extension $(2.22)$ and let $\Pi^{\prime}=\left\{\mathcal{H}^{\prime}, \Gamma_{0}^{\prime}, \Gamma_{1}^{\prime}\right\}$ be boundary triplet (2.24) for $S^{*}$. Assume that $\widetilde{A}:=\widetilde{S}_{\tau_{1}}$ (in the triplet $\left.\Pi^{\prime}\right)$. Since $\tau_{1} \in R_{u}\left[\mathcal{H}^{\prime}\right]$, it follows from Theorem 3.2 that $S(\widetilde{A})=S$. Moreover, according to Lemma $3.3 A \subset \widetilde{A}$ and $\widetilde{A}=\widetilde{A}_{\tau}$ (in the triplet $\Pi$ ), which proves the required statement.

Corollary 3.5. Let $\Pi=\left\{\mathcal{H}, \Gamma_{0}, \Gamma_{1}\right\}$ be a boundary triplet for $A^{*}$, let $A_{0}=\operatorname{ker} \Gamma_{0}$ and let $\tau \in \widetilde{R}_{c}(\mathcal{H})$. Then $S\left(\widetilde{A}_{\tau}\right) \cap A_{0}=A$ if and only if $\tau \in R_{c}[\mathcal{H}]$.

Proof. Assume that $\tau$ is represented as in (3.6). Then according to Proposition 3.4 $S\left(\widetilde{A}_{\tau}\right)=A_{\theta_{0}}$ with $\theta_{0}$ given by (2.21). Since obviously $A_{\theta_{0}} \cap A_{0}=A_{\widehat{\mathcal{K}}}$, the equivalences $S\left(\widetilde{A}_{\tau}\right) \cap A_{0}=A \Leftrightarrow \mathcal{K}=\{0\} \Leftrightarrow \tau \in R_{c}[\mathcal{H}]$ are valid.

In the following theorem we give a geometric characterization of self-adjoint extensions $\widetilde{A}_{\tau}$ corresponding to $\tau \in \widetilde{R}_{c}(\mathcal{H})$. 
Theorem 3.6. Assume that $\Pi=\left\{\mathcal{H}, \Gamma_{0}, \Gamma_{1}\right\}$ is a boundary triplet for $A^{*}$ and $A_{0}=$ ker $\Gamma_{0}$. Let $\tau \in \widetilde{R}(\mathcal{H})$ and let $\widetilde{A}_{\tau}$ be the corresponding exit space self-adjoint extension of A. Then:

(i) The linear relations $S\left(\widetilde{A}_{\tau}\right) \widehat{+} A_{0}$ and $T\left(\widetilde{A}_{\tau}\right)$ are closed if and only if $\tau \in \widetilde{R}_{c}(\mathcal{H})$.

(ii) $S\left(\widetilde{A}_{\tau}\right) \cap A_{0}=A$ and the linear relations $S\left(\widetilde{A}_{\tau}\right) \hat{+} A_{0}$ and $T\left(\widetilde{A}_{\tau}\right)$ are closed if and only if $\tau \in R_{c}[\mathcal{H}]$.

Proof. (i) We put $S=S\left(\widetilde{A}_{\tau}\right)$ and $T=T\left(\widetilde{A}_{\tau}\right)$. Assume that $\overline{S \widehat{+} A_{0}}=S \widehat{+} A_{0}$ and $\bar{T}=T$. Since $S \in \overline{\operatorname{ext}}(A)$ and $S \subset S^{*}$, it follows from Proposition 2.10, (ii) and (v) that $S=A_{\theta_{0}}$ with some symmetric relation $\theta_{0} \in \widetilde{\mathcal{C}}(\mathcal{H})$. Moreover, by Proposition 2.13 the decompositions

$$
\mathcal{H}=\mathcal{H}_{0} \oplus \mathcal{K}, \quad \theta_{0}=B \oplus \widehat{\mathcal{K}}
$$

holds with $\mathcal{K}=\operatorname{mul} \theta_{0}, \widehat{\mathcal{K}}=\widehat{\operatorname{mul}} \theta_{0}$ and $B \in \boldsymbol{B}\left(\operatorname{dom} \theta_{0}, \mathcal{H}_{0}\right)$, where $\operatorname{dom} \theta_{0} \subset \mathcal{H}_{0}$ is closed. Let $\mathcal{H}^{\prime \prime}=\operatorname{dom} \theta_{0}$ and $\mathcal{H}^{\prime}=\mathcal{H}_{0} \ominus \mathcal{H}^{\prime \prime}$, so that $\mathcal{H}_{0}=\mathcal{H}^{\prime} \oplus \mathcal{H}^{\prime \prime}$. Then decomposition (2.18) of $\mathcal{H}$ holds, the operator $B$ admits the block representation $(2.20)$ and $\theta_{0}$ can be written in the form (2.21). Moreover, since $B$ is symmetric, the equality $B_{2}=B_{2}^{*}$ holds. Therefore by Lemma 2.11, (ii) the equalities (2.24) define the boundary triplet $\Pi^{\prime}=\left\{\mathcal{H}^{\prime}, \Gamma_{0}^{\prime}, \Gamma_{1}^{\prime}\right\}$ for $S^{*}$. Since $\widetilde{A}=\widetilde{A}_{\tau}$ is an extension of $S$, it follows from Theorem 3.2 that $\widetilde{A}=\widetilde{A}_{\tau_{1}}$ (in the triplet $\Pi^{\prime}$ ) with some $\tau_{1} \in R_{u}\left[\mathcal{H}^{\prime}\right]$. Therefore by Lemma 3.3 $\tau \in \widetilde{R}_{c}(\mathcal{H})$.

Conversely, let $\tau \in \widetilde{R}_{c}(\mathcal{H})$. Then by Corollary 2.5 there exist a decomposition $(2.10)$ of $\mathcal{H}$, an operator $B=\left(B_{1}, B_{2}\right)^{\top} \in \boldsymbol{B}\left(\mathcal{H}^{\prime \prime}, \mathcal{H}^{\prime} \oplus \mathcal{H}^{\prime \prime}\right)$ and a function $\tau_{1} \in R_{u}\left[\mathcal{H}^{\prime}\right]$ such that $\tau=\left\{\mathcal{H}^{\prime} \oplus \mathcal{H}^{\prime \prime} \oplus \mathcal{K}, B_{1}, B_{2}, \tau_{1}\right\}$. It follows from Proposition 3.4 that $S=A_{\theta_{0}}$ with $\theta_{0}$ given by (2.21). Therefore by Proposition $2.13 \overline{S \widehat{+} A_{0}}=S \widehat{+} A_{0}$. Next assume that $\Pi^{\prime}=\left\{\mathcal{H}^{\prime}, \Gamma_{0}^{\prime}, \Gamma_{1}^{\prime}\right\}$ is a boundary triplet $(2.24)$ for $S^{*}$. Then by Lemma $3.3 \widetilde{A}_{\tau}=\widetilde{S}_{\tau_{1}}$ (in the triplet $\Pi^{\prime}$ ) and in view of Theorem 3.2 one has $\bar{T}=T$.

(ii) Combining statement (i) with Corollary 3.5 we arrive at statement (ii).

Proposition 3.7. Assume that $\Pi=\left\{\mathcal{H}, \Gamma_{0}, \Gamma_{1}\right\}$ is a boundary triplet for $A^{*}, \tau \in R_{u}[\mathcal{H}]$ and $\widetilde{A}_{\tau}=\widetilde{A}_{\tau}^{*}$ is the corresponding exit space extension of $A$. Let $\widetilde{A}_{\tau} \in \widetilde{\mathcal{C}}(\widetilde{\mathfrak{H}})$ with a Hilbert space $\widetilde{\mathfrak{H}} \supset \mathfrak{H}$ and let $\mathfrak{H}_{r}=\widetilde{\mathfrak{H}} \ominus \mathfrak{H}$. Then:

(i) There exist a symmetric relation $A_{r} \in \widetilde{\mathcal{C}}\left(\mathfrak{H}_{r}\right)$ and a boundary triplet $\Pi_{r}=\left\{\mathcal{H}, \Gamma_{0}^{r}, \Gamma_{1}^{r}\right\}$ for $A_{r}^{*}$ such that $\tau$ is the Weyl function of $\Pi_{r}$ and

$$
\widetilde{A}_{\tau}=\left\{\widehat{f} \oplus \widehat{f}_{r} \in A^{*} \oplus A_{r}^{*}: \Gamma_{0} \widehat{f}=\Gamma_{0}^{r} \widehat{f}_{r}, \Gamma_{1} \widehat{f}=-\Gamma_{1}^{r} \widehat{f}_{r}\right\} .
$$

(ii) $C\left(\widetilde{A}_{\tau}\right)=A_{-\mathcal{F}_{r}}$ (in the triplet $\Pi$ ), were $\mathcal{F}_{r}$ is the forbidden relation of $\Pi_{r}$.

Proof. Statement (i) directly follows from the results of $[6,8]$.

(i) According to Proposition 2.10, (ii) statement (ii) is equivalent to the equality

$$
C\left(\widetilde{A}_{\tau}\right)=\left\{\widehat{f} \in A^{*}:\left\{\Gamma_{0} \widehat{f},-\Gamma_{1} \widehat{f}\right\} \in \mathcal{F}_{r}\right\} .
$$

Let $\widehat{f}=\left\{f, f^{\prime}\right\} \in C\left(\widetilde{A}_{\tau}\right)$. Then $\widehat{f} \in A^{*}$ and by (3.3) there exists $f_{r}^{\prime} \in \mathfrak{H}_{r}$ such that $\widehat{g}:=\left\{f, f^{\prime} \oplus f_{r}^{\prime}\right\} \in \widetilde{A}_{\tau}$. Letting $\widehat{f}_{r}=\left\{0, f_{r}^{\prime}\right\} \in \mathfrak{H}_{r}^{2}$ one gets $\widehat{g}=\widehat{f} \oplus \widehat{f}_{r}$. Therefore $\widehat{f}_{r} \in A_{r}^{*}$ and hence $f_{r}^{\prime} \in \operatorname{mul} A_{r}^{*}$. Moreover, by (3.8) $\left\{\Gamma_{0} \widehat{f},-\Gamma_{1} \widehat{f}\right\}=\left\{\Gamma_{0}^{r} \widehat{f}_{r}, \Gamma_{1}^{r} \widehat{f}_{r}\right\} \in \mathcal{F}_{r}$.

Conversely, let $\widehat{f}=\left\{f, f^{\prime}\right\} \in A^{*}$ and $\left\{\Gamma_{0} \widehat{f},-\Gamma_{1} \widehat{f}\right\} \in \mathcal{F}_{r}$. Then there exists $f_{r}^{\prime} \in \operatorname{mul} A_{r}^{*}$ such that $\Gamma_{0}^{r}\left\{0, f_{r}^{\prime}\right\}=\Gamma_{0} \widehat{f}$ and $\Gamma_{1}^{r}\left\{0, f_{r}^{\prime}\right\}=-\Gamma_{1} \widehat{f}$. Letting $\widehat{f}_{r}=\left\{0, f_{r}^{\prime}\right\} \in A_{r}^{*}$ we obtain from (3.8) that $\widehat{g}:=\widehat{f} \oplus \widehat{f}_{r}=\left\{f, f^{\prime} \oplus f_{r}^{\prime}\right\} \in \widetilde{A}_{\tau}$ and therefore $\widehat{f}=\left\{f, f^{\prime}\right\} \in C\left(\widetilde{A}_{\tau}\right)$. This proves (3.9). 
In the following theorem we parameterize in terms of $\tau$ the compressions $C\left(\widetilde{A}_{\tau}\right)$ of exit space extensions $\widetilde{A}_{\tau}$ with $\tau \in \widetilde{R}_{c}(\mathcal{H})$.

Theorem 3.8. Assume that $\Pi=\left\{\mathcal{H}, \Gamma_{0}, \Gamma_{1}\right\}$ is a boundary triplet for $A^{*}, \tau \in \widetilde{R}_{c}(\mathcal{H})$, $\widetilde{A}_{\tau}=\widetilde{A}_{\tau}^{*}$ is the corresponding exit space extension of $A$ and $C\left(\widetilde{A}_{\tau}\right)$ is the compression of $\widetilde{A}_{\tau}$. Moreover, let $\tau_{0} \in R_{c}\left[\mathcal{H}_{0}\right]$ and $\mathcal{K}$ be the operator and multivalued parts of $\tau$ respectively (see (2.9)) and let $\mathcal{B}_{\tau_{0}} \in \boldsymbol{B}\left(\mathcal{H}_{0}\right)$ and $\mathcal{N}_{\tau_{0}}: \operatorname{dom} \mathcal{N}_{\tau_{0}} \rightarrow \mathcal{H}_{0}\left(\operatorname{dom} \mathcal{N}_{\tau_{0}} \subset \mathcal{H}_{0}\right)$ be operators corresponding to $\tau_{0}$ in accordance with Proposition 2.2. Then $C\left(\widetilde{A}_{\tau}\right)=A_{\theta_{c}}$ (in the triplet $\Pi$ ) with the symmetric linear relation $\theta_{c}$ in $\mathcal{H}$ given by

$$
\theta_{c}=-\mathcal{N}_{\tau_{0}}+\widehat{\operatorname{mul}} \theta_{c}=\left\{\left\{h,-\mathcal{N}_{\tau_{0}} h+h^{\prime}\right\}: h \in \operatorname{dom} \mathcal{N}_{\tau_{0}}, h^{\prime} \in \operatorname{mul} \theta_{c}\right\} .
$$

Moreover,

$$
\operatorname{ran} \mathcal{B}_{\tau_{0}} \oplus \mathcal{K} \subset \operatorname{mul} \theta_{c}, \quad \overline{\operatorname{mul} \theta_{c}}=\overline{\operatorname{ran} \mathcal{B}_{\tau_{0}}} \oplus \mathcal{K}
$$

If in addition ran $\mathcal{B}_{\tau_{0}}$ is closed, then $\overline{\text { mul } \theta_{c}}=\operatorname{mul} \theta_{c}=\operatorname{ran} \mathcal{B}_{\tau_{0}} \oplus \mathcal{K}$ and hence

$$
\theta_{c}=\left\{\left\{h,-\mathcal{N}_{\tau_{0}} h+\mathcal{B}_{\tau_{0}} \psi+k\right\}: h \in \operatorname{dom} \mathcal{N}_{\tau_{0}}, \psi \in \mathcal{H}_{0}, k \in \mathcal{K}\right\} .
$$

Proof. Assume that $\tau=\left\{\mathcal{H}^{\prime} \oplus \mathcal{H}^{\prime \prime} \oplus \mathcal{K}, B_{1}, B_{2}, \tau_{1}\right\}$ with $\mathcal{H}^{\prime} \oplus \mathcal{H}^{\prime \prime}=\mathcal{H}_{0}$ and $\tau_{1} \in R_{u}\left[\mathcal{H}^{\prime}\right]$ (see Corollary 2.5 and Remark 2.6). Moreover, let $\Gamma_{0}$ and $\Gamma_{1}$ have the block representations (2.19) and let $\theta_{0} \in \widetilde{\mathcal{C}}(\mathcal{H})$ be given by (2.21). Then according to Proposition $3.4 S=S\left(\widetilde{A}_{\tau}\right)$ admits the representation (2.22) and by Lemma 2.11, (ii) the equalities (2.24) define a boundary triplet $\Pi^{\prime}=\left\{\mathcal{H}^{\prime}, \Gamma_{0}^{\prime}, \Gamma_{1}^{\prime}\right\}$ for $S^{*}$. Moreover, according to Lemma $3.3 \widetilde{A}_{\tau}=\widetilde{S}_{\tau_{1}}$ (in the triplet $\Pi^{\prime}$ ) and by Proposition 3.7 there exist a Hilbert space $\mathfrak{H}_{r}$, a symmetric relation $S_{r} \in \widetilde{\mathcal{C}}\left(\mathfrak{H}_{r}\right)$ and a boundary triplet $\Pi_{r}=\left\{\mathcal{H}^{\prime}, \Gamma_{0}^{r}, \Gamma_{1}^{r}\right\}$ for $S_{r}^{*}$ such that $\tau_{1}$ is the Weyl function of $\Pi_{r}$ and $C\left(\widetilde{A}_{\tau}\right)=S_{-\mathcal{F}_{r}}$ (in the triplet $\Pi^{\prime}$ ), where $\mathcal{F}_{r}$ is the forbidden relation of $\Pi_{r}$. It follows from Theorem 2.19 that

$$
\begin{gathered}
\operatorname{ran} \mathcal{B}_{\tau_{1}} \subset \operatorname{mul} \mathcal{F}_{r}, \quad \overline{\operatorname{mul} \mathcal{F}_{r}}=\overline{\operatorname{ran} \mathcal{B}_{\tau_{1}}} \\
-\mathcal{F}_{r}=-\mathcal{N}_{\tau_{1}} \dot{+} \widehat{\operatorname{mul}} \mathcal{F}_{r}=\left\{\left\{h,-\mathcal{N}_{\tau_{1}} h+h^{\prime}\right\}: h \in \operatorname{dom} \mathcal{N}_{\tau_{1}}, h^{\prime} \in \operatorname{mul} \mathcal{F}_{r}\right\},
\end{gathered}
$$

where $\mathcal{N}_{\tau_{1}}: \operatorname{dom} \mathcal{N}_{\tau_{1}} \rightarrow \mathcal{H}^{\prime}$ is the operator given by

$$
\begin{gathered}
\operatorname{dom} \mathcal{N}_{\tau_{1}}=\left\{h \in \mathcal{H}^{\prime}: \lim _{y \rightarrow \infty} y \operatorname{Im}\left(\tau_{1}(i y) h, h\right)<\infty\right\} \\
\mathcal{N}_{\tau_{1}} h=\lim _{y \rightarrow \infty} \tau_{1}(i y) h, \quad h \in \operatorname{dom} \mathcal{N}_{\tau_{1}} .
\end{gathered}
$$

Let $\operatorname{dom} \theta_{c}^{\prime}:=\operatorname{dom} \mathcal{N}_{\tau_{1}} \oplus \mathcal{H}^{\prime \prime}$ and $\theta_{c}^{\prime}: \operatorname{dom} \theta_{c}^{\prime} \rightarrow \mathcal{H}_{0}$ be the operator given by

$$
\theta_{c}^{\prime}=\left(\begin{array}{cc}
-\mathcal{N}_{\tau_{1}} & B_{1} \\
B_{1}^{*}\left\lceil\operatorname{dom} \mathcal{N}_{\tau_{1}}\right. & B_{2}
\end{array}\right): \operatorname{dom} \mathcal{N}_{\tau_{1}} \oplus \mathcal{H}^{\prime \prime} \rightarrow \mathcal{H}^{\prime} \oplus \mathcal{H}^{\prime \prime}
$$

Then by Lemma $2.11 C\left(\widetilde{A}_{\tau}\right)=A_{\theta_{c}}$ (in the triplet $\Pi$ ) with the linear relation $\theta_{c}$ in $\mathcal{H}$ given by

$$
\begin{gathered}
\operatorname{mul} \theta_{c}=\operatorname{mul} \mathcal{F}_{r} \oplus \mathcal{K} \\
\theta_{c}=\theta_{c}^{\prime} \dot{+\operatorname{mul}} \theta_{c}=\left\{\left\{h, \theta_{c}^{\prime} h+h^{\prime}\right\}: h \in \operatorname{dom} \theta_{c}^{\prime}, h^{\prime} \in \operatorname{mul} \theta_{c}\right\}
\end{gathered}
$$

It follows from $(2.8)$ (with $\tau_{0}(\lambda)$ in place of $\left.\tau(\lambda)\right)$ that

$$
y \operatorname{Im}\left(\tau_{0}(i y) h, h\right)=y \operatorname{Im}\left(\tau_{1}(i y) P_{\mathcal{H}^{\prime}} h, P_{\mathcal{H}^{\prime}} h\right), \quad h \in \mathcal{H}_{0} .
$$

Therefore by (3.15) $\operatorname{dom} \theta_{c}^{\prime}=\operatorname{dom} \mathcal{N}_{\tau_{0}}$. Moreover, combining (3.17), (3.16) and (2.11) for each $h=h^{\prime} \oplus h^{\prime \prime} \in \operatorname{dom} \theta_{c}^{\prime}$ one obtains

$$
\begin{gathered}
\theta_{c}^{\prime} h=\left(-\mathcal{N}_{\tau_{1}} h^{\prime}+B_{1} h^{\prime \prime}\right) \oplus\left(B_{1}^{*} h^{\prime}+B_{2} h^{\prime \prime}\right)= \\
-\lim _{y \rightarrow \infty}\left(\left(\tau_{1}(i y) h^{\prime}-B_{1} h^{\prime \prime}\right) \oplus\left(-B_{1}^{*} h^{\prime}-B_{2} h^{\prime \prime}\right)\right)=-\lim _{y \rightarrow \infty} \tau_{0}(i y) h=-\mathcal{N}_{\tau_{0}} h .
\end{gathered}
$$


This and (3.19) yield (3.10).

Next, by $(2.11) \mathcal{B}_{\tau_{1}}=\mathcal{B}_{\tau_{0}} P_{\mathcal{H}^{\prime}}$ and hence $\operatorname{ran} \mathcal{B}_{\tau_{1}}=\operatorname{ran} \mathcal{B}_{\tau_{0}}$. Therefore by $(3.13)$ and (3.18) the relations (3.11) are valid. Finally, $\theta_{c}$ is symmetric in view Proposition 2.10, ( $\mathrm{v})$.

Theorem 3.9. Assume that $\Pi=\left\{\mathcal{H}, \Gamma_{0}, \Gamma_{1}\right\}$ is a boundary triplet for $A^{*}, A_{0}=\operatorname{ker} \Gamma_{0}$ (that is $A_{0}$ is a fixed canonical self-adjoint extension of $A$ in the Krein formula (2.36)), $\tau \in \widetilde{R}(\mathcal{H})$ and $\tau_{0} \in R\left[\mathcal{H}_{0}\right]$ is the operator part of $\tau$ (see (2.14)). Then the following statements are equivalent:

(i) $T\left(\widetilde{A}_{\tau}\right)$ is closed and $C\left(\widetilde{A}_{\tau}\right) \subset A_{0}$;

(ii) $\tau \in \widetilde{R}_{c}(\mathcal{H})$ and $\tau_{0}$ satisfies

$$
\lim _{y \rightarrow \infty} y \operatorname{Im}\left(\tau_{0}(i y) h, h\right)=\infty, \quad h \in \mathcal{H}_{0}, \quad h \neq 0 .
$$

If statement (i) (or equivalently (ii)) is valid then

$$
\overline{C\left(\widetilde{A}_{\tau}\right)}=\left\{\widehat{f} \in A^{*}: \Gamma_{0} \widehat{f}=0, \Gamma_{1} \widehat{f} \in \overline{\operatorname{ran} \mathcal{B}_{\tau_{0}}} \oplus \mathcal{K}\right\} .
$$

If in addition ran $\mathcal{B}_{\tau_{0}}$ is closed, then $C\left(\widetilde{A}_{\tau}\right)$ is closed and

$$
\text { (3.22) } C\left(\widetilde{A}_{\tau}\right)=\left\{\widehat{f} \in A^{*}: \Gamma_{0} \widehat{f}=0, \Gamma_{1} \widehat{f}=\mathcal{B}_{\tau_{0}} h \oplus k \text { with some } h \in \mathcal{H}_{0} \text { and } k \in \mathcal{K}\right\} \text {. }
$$

Proof. Assume statement (i). Then by (3.4) $S\left(\widetilde{A}_{\tau}\right) \subset A_{0}$ and hence $S\left(\widetilde{A}_{\tau}\right) \widehat{+} A_{0}=A_{0}$. Therefore $S\left(\widetilde{A}_{\tau}\right) \widehat{+} A_{0}$ is closed and by Theorem 3.6, (i) $\tau \in \widetilde{R}_{c}(\mathcal{H})$. This in view of Theorem 3.8 implies that $C\left(\widetilde{A}_{\tau}\right)=A_{\theta_{c}}$ with $\theta_{c}$ given by (3.10). It follows from (3.10) that $C\left(\widetilde{A}_{\tau}\right) \subset A_{0}$ if and only if $\operatorname{dom} \mathcal{N}_{\tau_{0}}=\{0\}$, which yields (3.20). Thus (ii) holds.

Conversely, let (ii) holds. Then by Theorem 3.6, (i) $T\left(\widetilde{A}_{\tau}\right)$ is closed. Moreover, by Theorem 3.8C $\left(\widetilde{A}_{\tau}\right)=A_{\theta_{c}}$ with $\theta_{c}$ of the form (3.10) and (3.20) shows that $\operatorname{dom} \mathcal{N}_{\tau_{0}}=$ $\{0\}$. Hence $\theta_{c}=\{0\} \oplus \operatorname{mul} \theta_{c}$ and therefore $C\left(\widetilde{A}_{\tau}\right) \subset A_{0}$, which yields statement (i). Moreover, by Proposition 2.10, (iv) $\overline{C\left(\widetilde{A}_{\tau}\right)}=A_{\bar{\theta}_{c}}$, where in view of $(3.11) \bar{\theta}_{c}=\{0\} \oplus$ $\overline{\operatorname{mul} \theta_{c}}=\{0\} \oplus \mathcal{H}_{c}$ with $\mathcal{H}_{c}=\overline{\operatorname{ran} \mathcal{B}_{\tau_{0}}} \oplus \mathcal{K}$. This yields (3.21). Finally, the last statement of the theorem follows from the previous one.

Corollary 3.10. Let the assumptions be the same as in Theorem 3.9. Then the following statements are equivalent:

(i) $T\left(\widetilde{A}_{\tau}\right)$ is closed and $\overline{C\left(\widetilde{A}_{\tau}\right)}=A_{0}$;

(ii) $\tau \in \widetilde{R}_{c}(\mathcal{H})$ and $\operatorname{ker} \mathcal{B}_{\tau_{0}}=\{0\}$.

If in addition $\operatorname{ran} \mathcal{B}_{\tau_{0}}$ is closed, then statement (ii) is equivalent to the following one:

(i') $T\left(\widetilde{A}_{\tau}\right)$ is closed and $C\left(\widetilde{A}_{\tau}\right)=A_{0}$.

Proof. Assume that (i) holds. Then by Theorem $3.9 \tau \in \widetilde{R}_{c}(\mathcal{H})$ and $\overline{C\left(\widetilde{A}_{\tau}\right)}=A_{\{0\} \oplus \mathcal{H}_{c}}$ with $\mathcal{H}_{c}=\overline{\operatorname{ran} \mathcal{B}_{\tau_{0}}} \oplus \mathcal{K}$. Since $A_{0}=A_{\{0\} \oplus \mathcal{H}}$, it follows from $\overline{C\left(\widetilde{A}_{\tau}\right)}=A_{0}$ that $\mathcal{H}_{c}=\mathcal{H}=$ $\mathcal{H}_{0} \oplus \mathcal{K}$. Hence $\overline{\operatorname{ran} \mathcal{B}_{\tau_{0}}}=\mathcal{H}_{0}$ or, equivalently, ker $\mathcal{B}_{\tau_{0}}=\{0\}$. Thus (ii) is valid.

Conversely, let (ii) holds. Then by Theorem 3.6, (i) $T\left(\widetilde{A}_{\tau}\right)$ is closed. Moreover, by Theorem $3.8 C\left(\widetilde{A}_{\tau}\right)=A_{\theta_{c}}$ with $\theta_{c}$ given by (3.10) and satisfying (3.11). Since $\overline{\text { ran } \mathcal{B}_{\tau_{0}}}=$ $\mathcal{H}_{0}$, it follows from (3.11) that $\overline{\operatorname{mul} \theta_{c}}=\mathcal{H}$. Hence by Lemma 2.7, (i) $\overline{\theta_{c}}=\{0\} \oplus \mathcal{H}$ and in view of Proposition 2.10, (iv) $\overline{C\left(\widetilde{A}_{\tau}\right)}=A_{\{0\} \oplus \mathcal{H}}=A_{0}$. This proves statement (i).

Assume now that ran $\mathcal{B}_{\tau_{0}}$ is closed. Then (i') implies (i) and, consequently, (ii). Conversely, let (ii) holds. Then (i) is valid and hence $C\left(\widetilde{A}_{\tau}\right)=A_{\theta_{c}}$ with $\theta_{c}=\{0\} \oplus \operatorname{mul} \theta_{c}$. Moreover, $\operatorname{ran} \mathcal{B}_{\tau_{0}}=\mathcal{H}_{0}$ and by Theorem 3.8 mul $\theta_{c}=\mathcal{H}_{0} \oplus \mathcal{K}=\mathcal{H}$. Therefore $C\left(\widetilde{A}_{\tau}\right)=A_{\{0\} \oplus \mathcal{H}}=A_{0}$, which yields $\left(\mathrm{i}^{\prime}\right)$. 
Corollary 3.11. Assume that $\Pi=\left\{\mathcal{H}, \Gamma_{0}, \Gamma_{1}\right\}$ is a boundary triplet for $A^{*}$ and $\tau \in$ $\widetilde{R}(\mathcal{H})$. Then $T\left(\widetilde{A}_{\tau}\right)$ is closed and $C\left(\widetilde{A}_{\tau}\right)=A$ if and only if $\tau \in R_{c}[\mathcal{H}], \mathcal{B}_{\tau}=0$ and

$$
\lim _{y \rightarrow \infty} y \operatorname{Im}(\tau(i y) h, h)=\infty, \quad h \in \mathcal{H}, \quad h \neq 0 .
$$

Proof. Let $T\left(\widetilde{A}_{\tau}\right)$ be closed and $C\left(\widetilde{A}_{\tau}\right)=A$. Then by Theorem $3.9 \tau \in \widetilde{R}_{c}(\mathcal{H})$, the operator-function $\tau_{0} \in R_{c}\left[\mathcal{H}_{0}\right]$ in decomposition (2.9) of $\tau$ satisfies (3.20) and $\overline{\operatorname{ran} \mathcal{B}_{\tau_{0}}} \oplus$ $\mathcal{K}=\{0\}$. Hence $\mathcal{K}=\{0\}$ and, consequently, $\mathcal{H}_{0}=\mathcal{H}$ and $\tau=\tau_{0}$. This implies that $\tau \in R_{c}[\mathcal{H}]$ and (3.23) holds. Moreover, $\operatorname{ran} \mathcal{B}_{\tau_{0}}=\{0\}$ and therefore $\mathcal{B}_{\tau}=\mathcal{B}_{\tau_{0}}=0$.

Conversely, let $\tau \in R_{c}[\mathcal{H}], \mathcal{B}_{\tau}=0$ and (3.23) is satisfied. Then by Theorem $3.9 T\left(\widetilde{A}_{\tau}\right)$ is closed and $\overline{C\left(\widetilde{A}_{\tau}\right)}$ is given by $(3.21)$ with $\operatorname{ran} \mathcal{B}_{\tau_{0}}=\operatorname{ran} \mathcal{B}_{\tau}=\{0\}$ and $\mathcal{K}=\{0\}$. Hence $C\left(\widetilde{A}_{\tau}\right)=\overline{C\left(\widetilde{A}_{\tau}\right)}=\operatorname{ker} \Gamma$ and by Proposition 2.10, (i) $C\left(\widetilde{A}_{\tau}\right)=A$.

Remark 3.12. Assume that $A$ is a closed densely defined symmetric operator in $\mathfrak{H}$. Then each exit space extension $\widetilde{A}=\widetilde{A}^{*}$ of $A$ is a densely defined operator and according to M.A. Naimark (see e.g. [1]) an extension $\widetilde{A}$ of $A$ is said to be of the second kind if $\operatorname{dom} \widetilde{A} \cap$ $\mathfrak{H}=\operatorname{dom} A$ or equivalently if $C(\widetilde{A})=A$. Clearly, in the case $\overline{\operatorname{dom} A}=\mathfrak{H}$ Corollary 3.11 gives a parametrisation of all extensions $\widetilde{A}$ of the second kind satisfying $\overline{T(\widetilde{A})}=$ $T(\widetilde{A})$. Note that this result follows from the results of [8], where a parametrisation of all extensions $\widetilde{A} \supset A$ of the second kind of a densely defined $A$ was obtained in terms of the parameter $\tau$ from the Krein formula (2.36). Observe also that a somewhat other parametrization of the second kind extensions can be found in $[22,25]$.

3.2. Exit space self-adjoint extensions with a self-adjoint compression. In the following proposition we provide a sufficient condition on the parameter $\tau$ for selfadjointness of the compression $C\left(\widetilde{A}_{\tau}\right)$.

Proposition 3.13. Assume that $\Pi=\left\{\mathcal{H}, \Gamma_{0}, \Gamma_{1}\right\}$ is a boundary triplet for $A^{*}, \tau \in \widetilde{R}_{c}(\mathcal{H})$ and $\tau_{0} \in R_{c}\left[\mathcal{H}_{0}\right]$ is the operator part of $\tau$ (see (2.9)). If $\operatorname{ran} \mathcal{B}_{\tau_{0}}$ is closed and

$$
\lim _{y \rightarrow \infty} y \operatorname{Im}\left(\tau_{0}(i y) h, h\right)<\infty, \quad h \in \operatorname{ker} \mathcal{B}_{\tau_{0}}
$$

then $C\left(\widetilde{A}_{\tau}\right)$ is self-adjoint.

Proof. Assume that ran $\mathcal{B}_{\tau_{0}}$ is closed and (3.24) holds. Then according to Theorem 3.8 $C\left(\widetilde{A}_{\tau}\right)=A_{\theta_{c}}$ with a symmetric linear relation $\theta_{c}$ in $\mathcal{H}$ given by (3.10) and satisfying $\overline{\operatorname{mul} \theta_{c}}=\operatorname{mul} \theta_{c}=\operatorname{ran} \mathcal{B}_{\tau_{0}} \oplus \mathcal{K}$. Moreover, by (3.24) $\operatorname{ker} \mathcal{B}_{\tau_{0}} \subset \operatorname{dom} \mathcal{N}_{\tau_{0}}=\operatorname{dom} \theta_{c}$. Therefore

$$
\mathcal{H} \ominus \operatorname{mul} \theta_{c}=\mathcal{H}_{0} \ominus \operatorname{ran} \mathcal{B}_{\tau_{0}}=\operatorname{ker} \mathcal{B}_{\tau_{0}} \subset \operatorname{dom} \theta_{c}
$$

and by Lemma 2.7, (ii) $\theta_{c}=\theta_{c}^{*}$. Now the required statement follows from Proposition $2.10,(\mathrm{v})$.

Corollary 3.14. Let the assumptions be the same as in Proposition 3.13. If $\operatorname{ran} \mathcal{B}_{\tau_{0}}$ is closed and there is a compact interval $[a, b] \subset \mathbb{R}$ such that $\tau_{0}$ admits a holomorphic continuation onto $\mathbb{R} \backslash[a, b]$, then $C\left(\widetilde{A}_{\tau}\right)$ is self-adjoint.

Proof. If the function $\tau_{0}$ is holomorphic in $\mathbb{C} \backslash[a, b]$, then it admits the Laurent expansion

$$
\tau_{0}(\lambda)=C_{0}+\lambda \mathcal{B}_{\tau_{0}}+\sum_{k=1}^{\infty} \frac{1}{\lambda^{k}} C_{k}, \quad|\lambda|>R>0
$$


with operator-valued coefficients $C_{k}=C_{k}^{*} \in \boldsymbol{B}\left(\mathcal{H}_{0}\right), k=0,1,2, \ldots$ Therefore

$$
\operatorname{Im}\left(\tau_{0}(i y) h, h\right)=\sum_{m=1}^{\infty} \frac{(-1)^{m}}{y^{2 m-1}}\left(C_{2 m-1} h, h\right), \quad y \in \mathbb{R}, \quad h \in \operatorname{ker} \mathcal{B}_{\tau_{0}}
$$

and,consequently,

$$
\lim _{y \rightarrow \infty} y \operatorname{Im}\left(\tau_{0}(i y) h, h\right)=-\left(C_{1} h, h\right)<\infty, \quad h \in \operatorname{ker} \mathcal{B}_{\tau_{0}} .
$$

Thus (3.24) holds and the required statement follows from Proposition 3.13.

In the following theorem we describe exit space self-adjoint extensions $\widetilde{A}$ of $A$ such that the compression $C(\widetilde{A})$ of $\widetilde{A}$ is self-adjoint and transversal with $A_{0}$.

Theorem 3.15. Assume the conditions of Theorem 3.9. Then the following statements are equivalent:

(i) $T\left(\widetilde{A}_{\tau}\right)$ is closed, $C\left(\widetilde{A}_{\tau}\right)^{*}=C\left(\widetilde{A}_{\tau}\right)$ and the extensions $C\left(\widetilde{A}_{\tau}\right)$ and $A_{0}$ are transversal;

(ii) $\tau \in R_{c}[\mathcal{H}]$ and

$$
\lim _{y \rightarrow \infty} y \operatorname{Im}(\tau(i y) h, h)<\infty, \quad h \in \mathcal{H} .
$$

Moreover, if (i) (equivalently (ii)) is satisfied, then

$$
C\left(\widetilde{A}_{\tau}\right)=A_{-\mathcal{N}_{\tau}}=\left\{\widehat{f} \in A^{*}: \Gamma_{1} \widehat{f}=-\mathcal{N}_{\tau} \Gamma_{0} \widehat{f}\right\},
$$

where $\mathcal{N}_{\tau} \in \boldsymbol{B}(\mathcal{H})$ is the operator given by

$$
\mathcal{N}_{\tau}=s-\lim _{y \rightarrow \infty} \tau(i y)
$$

Proof. Let statement (i) holds. Since $S\left(\widetilde{A}_{\tau}\right) \subset C\left(\widetilde{A}_{\tau}\right)$, it follows from Lemma 2.12 that the linear relation $S\left(\widetilde{A}_{\tau}\right) \hat{+} A_{0}$ is closed. Moreover, by (3.4) $S\left(\widetilde{A}_{\tau}\right) \cap A_{0}=A$. Therefore by Theorem 3.6, (ii) $\tau \in R_{c}[\mathcal{H}]$. Next, according to Theorem $3.8 C\left(\widetilde{A}_{\tau}\right)=A_{\theta_{c}}$, where $\theta_{c}$ is given by (3.10) with $\tau_{0}=\tau$, and by Proposition 2.10, (vii) $\theta_{c} \in \boldsymbol{B}(\mathcal{H})$. Therefore by (3.10) $\operatorname{dom} \mathcal{N}_{\tau}=\operatorname{dom} \theta_{c}=\mathcal{H}$, which in view of (2.2) yields (3.25). Thus statement (ii) is valid. Moreover, since mul $\theta_{c}=\{0\}$, it follows from (3.10) that $\theta_{c}=-\mathcal{N}_{\tau}$ and in view of definition (2.3) of $\mathcal{N}_{\tau}$ the equality (3.27) holds. This yields (3.26).

Conversely, assume (ii). Then by Theorem $3.6 T\left(\widetilde{A}_{\tau}\right)$ is closed. Moreover, by Theorem $3.8 C\left(\widetilde{A}_{\tau}\right)=A_{\theta_{c}}$, where $\theta_{c}$ is given by (3.10) with $\tau_{0}=\tau$ and (3.25) yields $\operatorname{dom} \theta_{c}=$ $\operatorname{dom} \mathcal{N}_{\tau}=\mathcal{H}$. Since $\theta_{c}$ is symmetric, this implies that $\theta_{c}=\theta_{c}^{*} \in \boldsymbol{B}(\mathcal{H})$. Therefore by Proposition 2.10, (v) and (vii) $C\left(\widetilde{A}_{\tau}\right)^{*}=C\left(\widetilde{A}_{\tau}\right)$ and the extensions $C\left(\widetilde{A}_{\tau}\right)$ and $A_{0}$ are transversal.

3.3. Finite-codimensional exit space extensions. Let $\widetilde{\mathfrak{H}} \supset \mathfrak{H}$ be a Hilbert space, let $\mathfrak{H}_{r}=\widetilde{\mathfrak{H}} \ominus \mathfrak{H}$ and let $\widetilde{A}=\widetilde{A}^{*} \in \widetilde{\mathcal{C}}(\widetilde{\mathfrak{H}})$ be an exit space extension of $A$. Such an extension is called finite-codimensional, if $n_{r}:=\operatorname{dim} \mathfrak{H}_{r}<\infty$.

The following proposition is well known (see e.g. [12]).

Proposition 3.16. Let $A \in \widetilde{\mathcal{C}}(\mathfrak{H})$ be a symmetric linear relation with finite deficiency indices $n_{+}(A)=n_{-}(A)=: d<\infty$ and let $\Pi=\left\{\mathcal{H}, \Gamma_{0}, \Gamma_{1}\right\}$ be a boundary triplet for $A^{*}$ (hence $\operatorname{dim} \mathcal{H}=d<\infty)$. Moreover, let $\tau \in \widetilde{R}(\mathcal{H})$ and let $\tau_{0} \in R\left[\mathcal{H}_{0}\right]$ be the operator part of $\tau$ (see (2.9)). Then $\widetilde{A}_{\tau}$ is finite-codimensional if and only if

$$
\tau_{0}(\lambda)=\mathcal{A}+\lambda \mathcal{B}+\sum_{j=1}^{l} \frac{1}{\alpha_{j}-\lambda} \mathcal{A}_{j}, \quad \lambda \in \mathbb{C} \backslash \mathbb{R}
$$


where

$$
\alpha_{j} \in \mathbb{R}, \quad \mathcal{A}, \mathcal{B}, \mathcal{A}_{j} \in \boldsymbol{B}\left(\mathcal{H}_{0}\right), \quad \mathcal{A}=\mathcal{A}^{*}, \mathcal{B} \geq 0, \mathcal{A}_{j} \geq 0, \mathcal{A}_{j} \neq 0, \quad j=1,2, \ldots, l .
$$

Moreover,

$$
n_{r}=\operatorname{dim} \operatorname{ran} \mathcal{B}+\sum_{j=1}^{l} \operatorname{dim} \operatorname{ran} \mathcal{A}_{j} .
$$

In the following two theorems we extend Proposition 3.16 to a ceratin class of selfadjoint extensions $\widetilde{A}$ of a symmetric relation $A$ with possibly infinite deficiency indices $n_{+}(A)=n_{-}(A) \leq \infty$ and characterise compressions $C(\widetilde{A})$ of such extensions.

Theorem 3.17. Let $A \in \widetilde{\mathcal{C}}(\mathfrak{H})$ be a symmetric linear relation in $\mathfrak{H}$ with equal deficiency indices $n_{+}(A)=n_{-}(A) \leq \infty$, let $\Pi=\left\{\mathcal{H}, \Gamma_{0}, \Gamma_{1}\right\}$ be a boundary triplet for $A^{*}$, let $A_{0}=\operatorname{ker} \Gamma_{0}$, let $\tau \in \widetilde{R}(\mathcal{H})$ and let $\widetilde{A}_{\tau}=\widetilde{A}_{\tau}^{*} \in \widetilde{\mathcal{C}}(\widetilde{\mathfrak{H}})(\widetilde{\mathfrak{H}} \supset \mathfrak{H})$ be the corresponding exit space extension of $A$ (see Theorem 2.20). Assume also that $\tau_{0} \in R\left[\mathcal{H}_{0}\right]$ is the operator part of $\tau$ (see (2.9)). Then the following statements are equivalent:

(i) $\widetilde{A}_{\tau}$ is finite-codimensional and the linear relation $S\left(\widetilde{A}_{\tau}\right) \widehat{+} A_{0}$ is closed;

(ii) $\tau_{0}$ is the rational function (3.28), (3.29) such that $\operatorname{dim} \operatorname{ran} \mathcal{B}<\infty$ and $\operatorname{dim} \operatorname{ran} A_{j}<$ $\infty, j=1,2, \ldots, l$.

Moreover, if statement (ii) (and hence (i)) is valid, then the dimension $n_{r}$ of $\mathfrak{H}_{r}=\widetilde{\mathfrak{H}} \ominus \mathfrak{H}$ satisfies (3.30).

Proof. Assume statement (i) and let $S_{r}=S_{r}\left(\widetilde{A}_{\tau}\right)$ and $T_{r}\left(\widetilde{A}_{\tau}\right)$ be linear relations in $\mathfrak{H}_{r}$ given by

$$
S_{r}=\widetilde{A}_{\tau} \cap \mathfrak{H}_{r}^{2}, \quad T_{r}\left(\widetilde{A}_{\tau}\right)=\left\{\left\{P_{\mathfrak{H}_{r}} f, P_{\mathfrak{H}_{r}} f^{\prime}\right\}:\left\{f, f^{\prime}\right\} \in \widetilde{A}_{\tau}\right\}
$$

Since $\operatorname{dim} \mathfrak{H}_{r}<\infty$, it follows that $T_{r}\left(\widetilde{A}_{\tau}\right)$ is closed and according to [7, Proposition 2.15] $T\left(\widetilde{A}_{\tau}\right)$ is closed as well. Therefore by Theorem $3.6 \tau \in \widetilde{R}_{c}(\mathcal{H})$. Let in accordance with Corollary $2.5 \tau_{0}$ be decomposed as in (2.11) with $\tau_{1} \in R_{u}\left[\mathcal{H}^{\prime}\right]$, that is $\tau=\left\{\mathcal{H}^{\prime} \oplus\right.$ $\left.\mathcal{H}^{\prime \prime} \oplus \mathcal{K}, B_{1}, B_{2}, \tau_{1}\right\}$ in the sense of Remark 2.6. Then by Proposition 3.4 the relation $S=S\left(\widetilde{A}_{\tau}\right)$ admits the representation $(2.22)$. Let $\Pi^{\prime}=\left\{\mathcal{H}^{\prime}, \Gamma_{0}^{\prime}, \Gamma_{1}^{\prime}\right\}$ be boundary triplet (2.24) for $S^{*}$. Then by Lemma $3.3 \widetilde{A}_{\tau}=\widetilde{S}_{\tau_{1}}$ (in the triplet $\Pi^{\prime}$ ). Moreover, according to [7, Lemma 2.14] $n_{ \pm}(S)=n_{ \pm}\left(S_{r}\right)$ and, consequently, $n_{+}(S)=n_{-}(S)<\infty$. Therefore $\operatorname{dim} \mathcal{H}^{\prime}<\infty$ and by Proposition 3.16

$$
\tau_{1}(\lambda)=\mathcal{A}_{0}+\lambda \mathcal{B}_{0}+\sum_{j=1}^{l} \frac{1}{\alpha_{j}-\lambda} \mathcal{A}_{0 j}, \quad \lambda \in \mathbb{C} \backslash \mathbb{R},
$$

where $\alpha_{j} \in \mathbb{R}$ and $\mathcal{A}_{0}, \mathcal{B}_{0}, \mathcal{A}_{0 j} \in \boldsymbol{B}\left(\mathcal{H}^{\prime}\right)$ are the operators satisfying $\mathcal{A}_{0}=\mathcal{A}_{0}^{*}, \mathcal{B}_{0} \geq 0$ and $\mathcal{A}_{0 j} \geq 0, \mathcal{A}_{0 j} \neq 0, j=1,2, \ldots, l$. This and the block representation $(2.11)$ of $\tau_{0}(\lambda)$ imply that $\tau_{0}(\lambda)$ is of the form (3.28) with

$$
\mathcal{A}=\left(\begin{array}{cc}
\mathcal{A}_{0} & -B_{1} \\
-B_{1}^{*} & -B_{2}
\end{array}\right), \quad \mathcal{B}=\left(\begin{array}{cc}
\mathcal{B}_{0} & 0 \\
0 & 0
\end{array}\right), \quad \mathcal{A}_{j}=\left(\begin{array}{cc}
\mathcal{A}_{0 j} & 0 \\
0 & 0
\end{array}\right), \quad j=1,2, \ldots, l .
$$

Hence $\mathcal{A}=\mathcal{A}^{*}, \mathcal{B} \geq 0, \mathcal{A}_{j} \geq 0, \mathcal{A}_{j} \neq 0$ and

$$
\operatorname{dim} \operatorname{ran} \mathcal{B}=\operatorname{dim} \operatorname{ran} \mathcal{B}_{0}<\infty, \quad \operatorname{dim} \operatorname{ran} \mathcal{A}_{j}=\operatorname{dim} \operatorname{ran} \mathcal{A}_{0 j}<\infty,
$$

which yields statement (ii). 
Conversely, let statement (ii) holds. Put $\varphi_{0}(\lambda)=\operatorname{Im} \lambda$ and $\varphi_{j}(\lambda)=\operatorname{Im} \frac{1}{\alpha_{j}-\lambda}$. Then by $(3.28)$

$$
\operatorname{Im} \tau_{0}(\lambda)=\varphi_{0}(\lambda) \mathcal{B}+\sum_{j=1}^{l} \varphi_{j}(\lambda) \mathcal{A}_{j}, \quad \lambda \in \mathbb{C} \backslash \mathbb{R} .
$$

Since $\operatorname{Im} \lambda \cdot \operatorname{Im} \varphi_{j}(\lambda)>0, j=0,1, \ldots, l$, it follows from (3.34) that

$$
\mathcal{H}^{\prime \prime}:=\operatorname{ker} \operatorname{Im} \tau_{0}(\lambda)=\operatorname{ker} \mathcal{B} \cap\left(\bigcap_{j=1}^{l} \operatorname{ker} \mathcal{A}_{j}\right)
$$

and, consequently, $\mathcal{H}^{\prime}:=\mathcal{H}_{0} \ominus \mathcal{H}^{\prime \prime}=\operatorname{ran} \mathcal{B}+\operatorname{ran} A_{1}+\cdots+\operatorname{ran} A_{l}$. Therefore $\operatorname{dim} \mathcal{H}^{\prime}<\infty$ and in view of (3.35) block representations (3.32) hold (with respect to the decomposition $\left.\mathcal{H}_{0}=\mathcal{H}^{\prime} \oplus \mathcal{H}^{\prime \prime}\right)$. Combining (3.32) with (3.28) one obtains the block representation (2.11) of $\tau_{0}(\lambda)$ with $\tau_{1} \in R\left[\mathcal{H}^{\prime}\right]$ given by (3.31). Since $\operatorname{dim} \mathcal{H}^{\prime}<\infty$, it follows that $\tau_{1} \in R_{u}\left[\mathcal{H}^{\prime}\right]$ and according to Proposition $2.3 \tau_{0} \in R_{c}\left[\mathcal{H}_{0}\right]$. Therefore $\tau \in \widetilde{R}_{c}(\mathcal{H})$ and by Theorem 3.6 $S\left(\widetilde{A}_{\tau}\right) \hat{+} A_{0}$ is closed.

Now it remains to show that $\widetilde{\mathcal{A}}_{\tau}$ is finite-codimensional and (3.30) holds. Since $\tau=$ $\left\{\mathcal{H}^{\prime} \oplus \mathcal{H}^{\prime \prime} \oplus \mathcal{K}, B_{1}, B_{2}, \tau_{1}\right\}$, it follows from Proposition 3.4 that $S=S\left(\widetilde{A}_{\tau}\right)$ admits the representation (2.22) and by Lemma 2.11 the equalities (2.24) define a boundary triplet $\Pi^{\prime}=\left\{\mathcal{H}^{\prime}, \Gamma_{0}^{\prime}, \Gamma_{1}^{\prime}\right\}$ for $S^{*}$. Therefore by Proposition $2.9 n_{ \pm}(S)=\operatorname{dim} \mathcal{H}^{\prime}<\infty$. Moreover, by Lemma $3.3 \widetilde{A}_{\tau}=\widetilde{S}_{\tau_{1}}$ (in the triplet $\Pi^{\prime}$ ). Thus in accordance with Proposition 3.16 $\widetilde{A}_{\tau}$ is finite-codimensional and

$$
n_{r}=\operatorname{dim} \operatorname{ran} \mathcal{B}_{0}+\sum_{j=1}^{l} \operatorname{dim} \operatorname{ran} \mathcal{A}_{0 j} .
$$

Combining this equality with (3.33) we arrive at (3.30).

Theorem 3.18. Let the assumptions of Theorem 3.17 be satisfied and let claim (ii) (equivalently (i)) of this theorem be valid. Moreover, let $\mathcal{A}^{\prime}=P_{\operatorname{ker}} \mathcal{B} \mathcal{A}\lceil\operatorname{ker} \mathcal{B} \in \boldsymbol{B}(\operatorname{ker} \mathcal{B})$, let $\mathcal{K}$ be the multivalued part of $\tau$ (see (2.9)) and let $\theta_{c}$ be a self-adjoint linear relation in $\mathcal{H}$ given by

$$
\theta_{c}=\left\{\left\{h,-\mathcal{A}^{\prime} h \oplus h^{\prime} \oplus k\right\}: h \in \operatorname{ker} \mathcal{B}, h^{\prime} \in \operatorname{ran} \mathcal{B}, k \in \mathcal{K}\right\}
$$

(this means that $-\mathcal{A}^{\prime}$ is the operator part of $\theta_{c}$ and $\left.\operatorname{mul} \theta_{c}=\operatorname{ran} \mathcal{B} \oplus \mathcal{K}\right)$. Then the extension $\widetilde{A}_{\tau}$ is finite-codimensional, the compression $C\left(\widetilde{A}_{\tau}\right)$ of $\widetilde{A}_{\tau}$ is self-adjoint and $C\left(\widetilde{A}_{\tau}\right)=A_{\theta_{c}}$ (in the triplet $\left.\Pi\right)$.

Moreover, the following holds:

(i) $C\left(\widetilde{A}_{\tau}\right)=A_{0}$ if and only if ker $\mathcal{B}=0$ (that is $\left.\mathcal{B}>0\right)$;

(ii) $C\left(\widetilde{A}_{\tau}\right)$ and $A_{0}$ are transversal if and only if $\tau \in R[\mathcal{H}]$ and $\mathcal{B}=0$, in which case $\theta_{c}=-\mathcal{A}$.

Proof. It follows from Theorem 3.17 that $\widetilde{A}_{\tau}$ is finite-codimensional. Moreover, by Theorem $3.8 C\left(\widetilde{A}_{\tau}\right)=\theta_{c}$ with symmetric relation $\theta_{c}$ in $\mathcal{H}$ given by (3.10). Since in view of (3.28)

$$
y \operatorname{Im}\left(\tau_{0}(i y) h, h\right)=y^{2}(\mathcal{B} h, h)+\sum_{j=1}^{l} \frac{y^{2}}{\alpha_{j}^{2}+y^{2}}\left(\mathcal{A}_{j} h, h\right), \quad h \in \mathcal{H}_{0},
$$


it follows that $\lim _{y \rightarrow \infty} y \operatorname{Im}\left(\tau_{0}(i y) h, h\right)<\infty$ if and only if $h \in \operatorname{ker} \mathcal{B}$. Therefore by definitions (2.2) and (2.3) $\operatorname{dom} \mathcal{N}_{\tau_{0}}=\operatorname{ker} \mathcal{B}$ and

$$
\mathcal{N}_{\tau_{0}} h=\lim _{y \rightarrow \infty} \tau_{0}(i y) h=\mathcal{A} h, \quad h \in \operatorname{ker} \mathcal{B}\left(=\operatorname{dom} \mathcal{N}_{\tau_{0}}\right)
$$

Moreover, by $(2.1) \mathcal{B}_{\tau_{0}}=\mathcal{B}$ and the finite-dimensionality of $\operatorname{ran} \mathcal{B}$ yields $\operatorname{ran} \mathcal{B}_{\tau_{0}}=$ $\overline{\operatorname{ran} \mathcal{B}_{\tau_{0}}}=\operatorname{ran} \mathcal{B}$. Therefore by Theorem $3.8 \operatorname{mul} \theta_{c}=\operatorname{ran} \mathcal{B} \oplus \mathcal{K}$ and (3.10) can be written as

$$
\theta_{c}=\left\{\left\{h,-\mathcal{A} h+\left(h^{\prime} \oplus k\right)\right\}: h \in \operatorname{ker} \mathcal{B}, h^{\prime} \in \operatorname{ran} \mathcal{B}, k \in \mathcal{K}\right\}
$$

Since $\mathcal{H}_{0}=\operatorname{ker} \mathcal{B} \oplus \operatorname{ran} \mathcal{B}$ and $h^{\prime} \in \operatorname{ran} \mathcal{B}$ in (3.37) is arbitrary, the equality (3.37) is equivalent to (3.36). Finally, statements (i) and (ii) are implied by Corollary 3.10 and Theorem 3.15.

3.4. The case of finite deficiency indices. In this subsection we suppose that the following assumption (a) is satisfied:

(a) $A$ is a closed symmetric linear relation in $\mathfrak{H}$ with finite deficiency indices $n_{+}(A)=$ $n_{-}(A)<\infty, \Pi=\left\{\mathcal{H}, \Gamma_{0}, \Gamma_{1}\right\}$ is a boundary triplet for $A^{*}, \tau \in \widetilde{R}(\mathcal{H}), \tau_{0} \in R\left[\mathcal{H}_{0}\right]$ is the operator part of $\tau$ (see $(2.9)), \widetilde{A}_{\tau}=\widetilde{A}_{\tau}^{*}$ is the corresponding exit space extension of $A$ and $C\left(\widetilde{A}_{\tau}\right)$ is the compression of $\widetilde{A}_{\tau}$.

Then $\operatorname{ext}(A)=\overline{\operatorname{ext}}(A)$ and hence for each exit space extension $\widetilde{A}=\widetilde{A}^{*}$ of $A$ the linear relations $C(\widetilde{A}), T(\widetilde{A})$ and $S(\widetilde{A}) \hat{+} A_{0}$ are closed (here $A_{0}=\operatorname{ker} \Gamma_{0}$ ). Moreover, under the assumption (a) $\operatorname{dim} \mathcal{H}<\infty$, which implies that $\widetilde{R}(\mathcal{H})=\widetilde{R}_{c}(\mathcal{H}), R\left[\mathcal{H}^{\prime}\right]=R_{c}\left[\mathcal{H}^{\prime}\right]$ and $\overline{\operatorname{ran} \mathcal{B}_{\tau}}=\operatorname{ran} \mathcal{B}_{\tau}$ for any subspace $\mathcal{H}^{\prime} \subset \mathcal{H}$ and operator-function $\tau \in R\left[\mathcal{H}^{\prime}\right]$. These facts together with Theorems 3.8, 3.9 and Corollaries 3.10, 3.11 yield the following two theorems.

Theorem 3.19. Let the assumption (a) be satisfied. Then $C\left(\widetilde{A}_{\tau}\right)=A_{\theta_{c}}$ (in the triplet П) with the symmetric linear relation $\theta_{c}$ in $\mathcal{H}$ defined by (3.12).

Theorem 3.20. Let the assumption (a) be satisfied and let $A_{0}=\operatorname{ker} \Gamma_{0}$. Then:

(i) $C\left(\widetilde{A}_{\tau}\right) \subset A_{0}$ if and only if the operator function $\tau_{0}$ satisfies

$$
\lim _{y \rightarrow \infty} y \operatorname{Im}\left(\tau_{0}(i y) h, h\right)=\infty, \quad h \in \mathcal{H}_{0}, \quad h \neq 0 .
$$

In this case $C\left(\widetilde{A}_{\tau}\right)$ is defined by abstract boundary conditions (3.22).

(ii) $C\left(\widetilde{A}_{\tau}\right)=A_{0}$ if and only if ker $\mathcal{B}_{\tau_{0}}=0$ (that is $\mathcal{B}_{\tau_{0}}>0$ ).

(iii) $C\left(\widetilde{A}_{\tau}\right)=A$ if and only if $\tau \in R[\mathcal{H}], \mathcal{B}_{\tau}=0$ and

$$
\lim _{y \rightarrow \infty} y \operatorname{Im}(\tau(i y) h, h)=\infty, \quad h \in \mathcal{H}, \quad h \neq 0 .
$$

In the following theorem we describe all exit space extensions $\widetilde{A}=\widetilde{A}^{*}$ of a symmetric relation $A$ with finite deficiency indices such that the compression $C(\widetilde{A})$ of $\widetilde{A}$ is selfadjoint.

Theorem 3.21. Let the assumption (a) be satisfied and let $A_{0}=\operatorname{ker} \Gamma_{0}$. Then:

(i) $C\left(\widetilde{A}_{\tau}\right)$ is self-adjoint if and only if

$$
\lim _{y \rightarrow \infty} y \operatorname{Im}\left(\tau_{0}(i y) h, h\right)<\infty, \quad h \in \operatorname{ker} \mathcal{B}_{\tau_{0}} .
$$

If in particular $\tau_{0}$ is holomorphic out of a compact interval $[a, b] \subset \mathbb{R}$, then $C\left(\widetilde{A}_{\tau}\right)$ is self-adjoint. 
(ii) $C\left(\widetilde{A}_{\tau}\right)$ is self-adjoint and transversal with $A_{0}$ if and only if $\tau \in R[\mathcal{H}]$ and

$$
\lim _{y \rightarrow \infty} y \operatorname{Im}(\tau(i y) h, h)<\infty, \quad h \in \mathcal{H} .
$$

In this case

$$
C\left(\widetilde{A}_{\tau}\right)=A_{-\mathcal{N}_{\tau}}=\left\{\widehat{f} \in A^{*}: \Gamma_{1} \widehat{f}=-\mathcal{N}_{\tau} \Gamma_{0} \widehat{f}\right\},
$$

where $\mathcal{N}_{\tau}=\mathcal{N}_{\tau}^{*} \in \boldsymbol{B}(\mathcal{H})$ is the operator given by $\mathcal{N}_{\tau}=\lim _{y \rightarrow \infty} \tau(i y)$.

Proof. (i) According to Theorem $3.19 C\left(\widetilde{A}_{\tau}\right)=A_{\theta_{c}}$ with a symmetric linear relation $\theta_{c}$ in $\mathcal{H}$ defined by (3.12). It follows from (3.12) that mul $\theta_{c}=\operatorname{ran} \mathcal{B}_{\tau_{0}} \oplus \mathcal{K}$ and therefore $\mathcal{H} \ominus \operatorname{mul} \theta_{c}=\mathcal{H}_{0} \ominus \operatorname{ran} \mathcal{B}_{\tau_{0}}=\operatorname{ker} \mathcal{B}_{\tau_{0}}$. Moreover by (3.12) $\operatorname{dom} \theta_{c}=\operatorname{dom} \mathcal{N}_{\tau_{0}}$ and hence the inclusion $\mathcal{H} \ominus \operatorname{mul} \theta_{c} \subset \operatorname{dom} \theta_{c}$ is equivalent to (3.40). Therefore by Lemma 2.7, (iii) (3.40) is equivalent to self-adjointness of $\theta_{c}$, which by Proposition 2.10, (v) is equivalent to $C\left(\widetilde{A}_{\tau}\right)^{*}=C\left(\widetilde{A}_{\tau}\right)$. Thus $C\left(\widetilde{A}_{\tau}\right)^{*}=C\left(\widetilde{A}_{\tau}\right)$ if and only if $(3.40)$ holds. The second part of the statement (i) is implied by Corollary 3.14.

(ii) Statement (ii) directly follows from Theorem 3.15 and arguments before Theorem 3.19 .

In the following theorem we characterize compressions of finite-codimensional extensions $\widetilde{A}=\widetilde{A}^{*}$ of a symmetric relation $A$ with finite deficiency indices. The statements of this theorem follow from Theorem 3.18 and arguments before Theorem 3.19.

Theorem 3.22. Let under the assumption (a) $\tau_{0}$ be a rational function (3.28), (3.29) (according to Proposition 3.16 this assumption is equivalent to the finite-codimensionality of $\widetilde{A}_{\tau}$ ). Moreover, let $\mathcal{A}^{\prime}=P_{\operatorname{ker} \mathcal{B}} \mathcal{A}\lceil\operatorname{ker} \mathcal{B}$ and let $\mathcal{K}$ be the multivalued part of $\tau$ (see (2.9)). Then $C\left(\widetilde{A}_{\tau}\right)$ is a self-adjoint linear relation in $\mathfrak{H}$ and $C\left(\widetilde{A}_{\tau}\right)=A_{\theta_{c}}$ with the relation $\theta_{c}=\theta_{c}^{*} \in \widetilde{\mathcal{C}}(\mathcal{H})$ given by (3.36). Moreover, statements (i) and (ii) of Theorem (3.18) are valid.

Corollary 3.23. Let $\widetilde{\mathfrak{H}}$ be a Hilbert space, let $\mathfrak{H}$ be a subspace of a finite codimension in $\widetilde{\mathfrak{H}}$ (this means that $\operatorname{dim}(\widetilde{\mathfrak{H}} \ominus \mathfrak{H})<\infty)$ and let $\widetilde{A}=\widetilde{A}^{*}$ be a linear relation in $\widetilde{\mathfrak{H}}$. Then the compression $C(\widetilde{A})=P_{\mathfrak{H}} \widetilde{A} \uparrow \mathfrak{H}$ of $\widetilde{A}$ (see $(3.3)$ ) is a self-adjoint linear relation in $\mathfrak{H}$.

Proof. Clearly, $\widetilde{A}$ is an exit space extension of the symmetric relation $S=S(\widetilde{A})$ (see (3.1)). Moreover, it was shown in the proof of Theorem 3.17 that $n_{+}(S)=n_{-}(S)<\infty$. Therefore by Theorem $3.22 C(\widetilde{A})$ is self-adjoint.

Remark 3.24. Statement of Corollary 3.23 was proved by another method in [3] (for the case of an operator $\widetilde{A}=\widetilde{A}^{*}$ see $\left.[24]\right)$.

\section{REFERENCES}

[1] N. I. Akhiezer, I. M.Glazman, Theory of linear operators in Hilbert space, vol. I and II, Pitman, Boston-London-Melbourne, 1981.

[2] T.Ya. Azizov, A. Dijksma, Closedness and adjoints of products of operators, and compressions, Int. Equ. Oper. Theory 74 (2012), 259-269.

[3] T.Ya. Azizov, A. Dijksma, and G. Wanjala, Compressions of maximal dissipative and self-adjoint linear relations and of dilations, Linear Algeb. Appl. 439 (2013), 771- 792.

[4] T.Ya. Azizov, B. Ćurgus, and A. Dijksma, Finite-codimensional compressions of symmetric and self-adjoint linear relations in Krein spaces, Int. Equ. Oper. Theory 86 (2016), 71-95.

[5] V. M. Bruk, Extensions of symmetric relations, Math. Notes 22 (1977), no.6, 953-958.

[6] V.A. Derkach, S. Hassi, M.M. Malamud, and H.S.V. de Snoo, Generalized resolvents of symmetric operators and admissibility, Methods of Functional Analysis and Topology 6 (2000), no. 3, 24-55.

[7] V. A. Derkach, S. Hassi, M. Malamud, and H. S.V. de Snoo,Boundary Relations and their Weyl families, Trans. Amer. Math. Soc. 358 (2006), no. 12, 5351-5400. 
[8] V.A. Derkach, S. Hassi, M.M. Malamud, and H.S.V. de Snoo, Boundary relations and generalized resolvents of symmetric operators, Russian J. Math. Ph. 16 (2009), no. 1, 17-60.

[9] V.A. Derkach and M.M. Malamud, Generalized resolvents and the boundary value problems for Hermitian operators with gaps, J. Funct. Anal. 95 (1991),1-95.

[10] V.A. Derkach and M.M. Malamud, The extension theory of Hermitian operators and the moment problem, J. Math. Sciences 73 (1995), no. 2, 141-242.

[11] A. Dijksma and H. Langer, Finite-dimensional self-adjoint extensions of a symmetric operator with finite defect and their compressions, In: Advances in complex analysis and operator theory, Festschrift in honor of Daniel Alpay, Birkhäuser, Basel, (2017), 135-163.

[12] A. Dijksma and H. Langer, Compressions of self-adjoint extensions of a symmetric operator and M.G. Kreins resolvent formula, Integr. Equ. Oper. Theory 90:41 (2018).

[13] A. Dijksma and H.S.V. de Snoo, Self-adjoint extensions of symmetric subspaces, Pasif. J. Math. 54 (1974), no. 1, 71-100.

[14] V.I. Gorbachuk and M.L. Gorbachuk, Boundary problems for differential-operator equations, Kluver Acad. Publ., Dordrecht-Boston-London, 1991. (Russian edition: Naukova Dumka, Kiev, 1984).

[15] M.G. Krein and H. Langer,On defect subspaces and generalized resolvents of a Hermitian operator in the space $\Pi_{\kappa}$, Funct. Anal. Appl. 5 (1971/1972), 136-146, 217-228

[16] M.G Krein and H. Langer, Über die Q-functions eines $\pi$-hermiteschen operators in raume $\Pi_{\kappa}$, Acta. Sci. Math. (Szeged) 34 (1973), 191-230.

[17] H. Langer and B. Textorious, On generalized resolvents and Q-functions of symmetric linear relations (subspaces) in Hilbert space, Pacif. J. Math. 72(1977), no. 1, 135-165.

[18] M. M. Malamud, On the formula of generalized resolvents of a nondensely defined Hermitian operator, Ukr. Math. Zh. 44(1992), no. 12, 1658-1688.

[19] B. Sz.-Nagy, C. Foias, Harmonic Analysis of Operators in Hilbert Space, Paris and Akad..Kiado, Budapest, 1967.

[20] M.A. Nudelman, A generalization of Stengers lemma to maximal dissipative operators. Integr. Equ. Oper. Theory 70 (2011), 301-305.

[21] A.V.Shtraus, On selfadjoint operators in the orthogonal sum of Hilbert spaces, Dokl. Akad. Nauk SSSR, 144 (1962), no.3, 512-515.

[22] A. V. Shtraus, On one-parameter families of extensions of a symmetric operator, Izv. Akad. Nauk SSSR, Ser. Mat. 30 (1966), 1325-1352.

[23] A. V. Shtraus, Extensions and generalized resolvents of a symmetric operator which is not densely defined, Izv. Akad. Nauk SSSR. Ser. Mat. 34 (1970),no.1, 175-202. (Russian); English translation: Mathematics of the USSR-Izvestiya, 4 (1970), no. 1, 179-208.

[24] W. Stenger, On the projection of a selfadjoint operator, Bull. Am. Math. Soc. 74(1968), 369-372.

[25] S.M. Zagorodnyuk, Generalized resolvents of symmetric and isometric operators: the Shtraus approach, Ann. Funct. Anal. 4 (2013), no. 1, 175-285.

Department of Mathematical Analysis and Informatics, Poltava National V.G. Korolenko Pedagogical University, Ostrogradski Str. 2, 36000 Poltava, Ukraine

E-mail address: vadim.mogilevskii@gmail.com 